Working Paper

Economic Series 10-28

September 2011
Departamento de Economía

Universidad Carlos III de Madrid

Calle Madrid, 126

28903 Getafe (Spain)

Fax (34) 916249875

\title{
Heterogeneous Tiebout Communities with Private Production and Anonymous Crowding ${ }^{*}$
}

\author{
Jaime Luque \\ Departamento de Economía \\ Universidad Carlos III de Madrid \\ japluque@eco.uc3m.es
}

\begin{abstract}
This paper examines, in the context of a multiple types of consumers, a set of necessary and sufficient conditions under which equilibrium and optimum exist, and involve mixing types of consumers in jurisdictions. Pricing includes visa permits for entry. Following Berglas (1976), we assume anonymous crowding and complementarities in production. For a large economy, we prove existence of equilibrium and the first and second welfare theorems. Our simultaneous optimization approach provides a new technique for showing existence of equilibrium in local public good economies with local production and a continuum of agents.
\end{abstract}

JEL Classification: C70, D71, H40, R13.

Keywords and Phrases: local public goods; collaborative production; wages; anonymous crowding; visa permits; societal stratification; heterogeneous Tiebout communities; generalized game.

* A different version of this paper circulated under the title "Where to live? Wages, community composition and public goods". The paper was presented at EWGE 2010 (Kracow), APET 2009 (Galway), PGPPE 2008 Workshop (Bonn), ASSET 2008 (Florence) and the European Winter Meeting of the Econometric Society 2007 (Brussels). I thank the comments of these audiences, and especially N. Allouch, M. Berliant, K. Desmet, P. Gottardi, R. Feldman, H. Konishi, M. Páscoa, E. Moreno, and M. Wooders. The author gratefully acknowledges the financial support of FCT and INOVA (Portugal), the European Science Foundation (through the activity "Public Goods, Public Projects and Externalities"), and the Spanish Ministry of Education and Science under the grant SEJ2008-03516. ADDRESS: Departamento de Economia, Universidad Carlos III de Madrid; Calle Madrid 126, 28903 Getafe (Madrid), SPAIN. 


\section{Introduction}

Tiebout (1956) claimed that in a model with local public goods and many jurisdictions (among other conditions), equilibrium will feature sorting of consumers by type and that the resulting allocation will be efficient. Twenty years later, Berglas (1976) proposed a frictionless production Tiebout economy, where private goods are produced within each community by means of its labor inputs, drop-

ping in this way the unnecessary and unrealistic assumption of "no restrictions due to employment opportunities" from the Tiebout model. In an anonymous crowding scenario (the consumers care only about the level of congestion of the public goods and not about the identities of the other individuals making use of them), Berglas showed that if individuals differ in their productive skills (teachers, accountants, unskilled workers, etc.), the distributions of tastes for public goods and labor skills are independent, and labor skills in a community are complementary, then individuals may become better off forming mixed communities rather than sorting into homogeneous communities.

Berglas' purpose was to analyze the formation of mixed communities, its efficiency, and the associated tax structures. However, Berglas (1976) did not demonstrate existence of equilibrium and his existence conjecture was subject to Bewley's (1981) criticism. The issue of the existence of equilibrium was also left aside in the subsequent literature that analyze the formation of mixed communities (see, for example, Bartolome (1990), Benabou (1993), McGuire (1991), and Schwab and Oates (1991)). Those models suffer from several shortcomings that prevent the study of the existence of equilibrium. In particular, the approach through differential techniques is inappropriate when considering the population and locations as discrete sets. This well known "integer problem" was first analyzed by Pauly (1970) and Wooders (1978), and concisely summarized by Starrett (1988). Proving existence of equilibrium is a fundamental step that must be done before aiming at any normative and empirical test of the model. Otherwise, equilibrium may fail to exist, suggesting that the equations that describe the model are not consistent with each other.

Being inspired by Berglas' (1976) work, we propose an alternative general equilibrium model that incorporates Berglas' main assumptions: anonymous crowding, different labor skills among individual types, and labor complementarities in the production of private goods. Although our model is different from Berglas (1976), we recognize his pioneering work on this subject, and for this reason, 
hereinafter refer to our economy as a Berglas economy. The extension of Berglas (1976) to a jurisdictions economy hinges on a conception of how the world is articulated: jurisdictions are "small" in size. Negligible jurisdictions are modeled following the works of Allouch, Conley, and Wooders (2009) (hereinafter, ACW) and Ellickson, Grodal, Scotchmer, and Zame (1999) (hereinafter EGSZ). We consider a world whose population splits into city districts, municipalities, villages, and counties, and not into countries with a "big" size. ${ }^{1}$

For the Berglas economy proposed here, our main formal goal is to demonstrate that heterogeneous Tiebout communities exist and are optimal in equilibrium. To obtain this result we need to identify the conditions under which 1) equilibrium exists, 2) the set of equilibria is equivalent to the core, and 3) the core involves mixing types of consumers in jurisdictions. Our descriptive and normative analysis of heterogeneous mixed communities is important in the following respects. First, the analysis refutes Bewley's (1981, p. 725, 733) argument against the possibility of extending Tiebout's analysis to include heterogeneous communities. Second, only by bringing job opportunities into Tiebout's model can we properly understand the pricing characteristics of a market of visa permits. $^{2}$ Third, we demonstrate that Tiebout's theory does not speak against the empirical evidence that analyzes heterogeneous communities in places that offer both public goods and industries to work with (see, for example, Roback (1982)).

Our proof of existence of equilibrium is novel, different from the previous core decentralization approach (see Wooders (1978), Conley and Wooders (1997), and ACW (2009)) and the non-excess demand approach (see EGSZ (1999)). Here, we follow a simultaneous optimization approach. For this, we construct a generalized game for our atomless local public goods and production economy, prove that this game has an equilibrium in pure strategies, and then show that such equilibrium is, in fact, a price taking equilibrium for a Berglas economy. This proof constitutes by itself a contribution to the clubs / local public goods literature. For pedagogical reasons, we explain in Section 5 the difference between these three approaches and also the new subtleties that this third approach has for these types of economies (local public goods, continuum of consumers, purifi-

\footnotetext{
${ }^{1}$ Big cities such as London or New York are thought of as conglomerates of jurisdictions, such as Knightsbridge (London) or Soho (New York). Both are clear examples of jurisdictions with heterogeneous consumers (heterogeneity in their income levels, productive skills and preferences).

${ }^{2}$ Some countries, such as the U.K., are considering selling visa permits to enter the country to work there.
} 
cation of equilibrium).

The literature on community composition coming after Tiebout's tale has been extensive. It is well established (Scotchmer and Wooders (1987)) that communities should be taste-homogenous if crowding types are exogenous and crowding is anonymous. Non-anonymous crowding (consumers have preferences for the other types of consumers with whom they wish to share a jurisdiction) allowed Alesina and La Ferrara (2000), ACW (2009), and EGSZ (1999, 2006) to recover heterogeneity in the community composition. We depart from these papers in that we show that heterogeneous communities exist and are optimal in a context of anonymous crowding and exogenous crowding types if a local collaborative production technology is added into the picture.

To our knowledge, Conley and Wooders (2001) and Konishi (2010) are the only exceptions in the theoretical literature that obtains optimal taste-heterogeneous jurisdictions in a context of anonymous crowding. However, both models differ from the present one. On the one hand, Conley and Wooders (2001) obtain heterogeneity in community composition through different agents' genetic endowments and endogenous crowding types (through educational choices). Although these authors discuss the need to drop Tiebout's assumption of "no restriction due to labor opportunities", their model does not incorporate a private production sector associated with a jurisdiction, nor does it touch the point of how labor complementarities induce agents to sort into heterogeneous communities. Konishi (2010), on the other hand, addresses the issue of existence of heterogeneous clubs in a context of anonymous crowding. However, even if his result seems similar to ours, the source of heterogeneity is totally different. There, mixed clubs result and are efficient if clubs have multiple facilities (e.g., gym and swimming pool) with economies of scope, whereas in the present paper heterogeneous communities arise due to the labor complementarities in the production of a jurisdiction industry.

Our model captures the "group composition problem" pointed out by Berglas (1976). In our setting, each jurisdiction offers both public goods and a specific industry. ${ }^{3}$ Therefore, wages are both type and jurisdiction specific. This implies that the jurisdiction-type specific wage rate results in different wages among

\footnotetext{
${ }^{3}$ The comparison between jurisdictions with different production technologies and supply of public goods captures the profound debate in the economic policy arena: more versus less market oriented economies, e.g., Silicon Valley (California, U.S.A.) versus Keilaniemi, the basement of Nokia (Espoo, Finland), respectively.
} 
the different individuals of the jurisdiction. This treatment of production differs from Wooders (1978), who makes production dependent only on the size of the jurisdiction. Also, the assumption of a jurisdiction specific industry departs from Benabou (1993), who models production as a citywide activity (same industry for several jurisdictions). We introduce no contractual problems into the model (skills are observable and adverse selection problems are ruled out). ${ }^{4}$ Our attention is primarily oriented toward the properties of societal stratification.

Another related paper is Konishi (2008), which models a Tiebout economy with entrepreneurs, where communities have an associated exogenous wage in each jurisdiction. In that paper, communities are homogeneously populated as a consequence of the imposition of a zoning constraint that makes crowding effects anonymous. In our paper we take the standard assumption of a complete price system for the set of jurisdiction types (thus, entrepreneurs are absent). We also depart from Konishi (2008) in that we make the production technology and wages endogenously dependent on the profile of worker types in a jurisdiction. This further step is crucial for the study of the different possible patterns of community composition. The idea is that if the private production in a jurisdiction is collaborative (e.g., Leontieff or Cobb-Douglas) and pays high wages, then the individuals will prefer to group into a heterogeneous community, once the pattern of community composition fits with the labor complementarities required in the production sector. This result holds true in an anonymous crowding framework.

Our work also bears some relationship to that of EGSZ (2006), where social interaction externalities are embedded in a general equilibrium model: the membership pricing of the group formation (inputs and outputs) accommodates the existing externalities within a firm, such as poor working conditions and uncongenial co-workers. EGSZ (2006) give many interesting examples, but do not demonstrate equilibrium existence and core decentralization in this new model (the authors highlight only some new difficulties compared with EGSZ's (1999) proofs). Our model differs from EGSZ (2006) in that we consider a scenario with anonymous crowding, and therefore, externalities within a community of the types mentioned above do not enter into consumers' preferences, and therefore, are not priced. Another difference is that we explicitly consider a production technology associated with each jurisdiction that exhibits complementarities in its labor inputs. By assuming that the production technology exhibits constant returns to scale, we are able to parameterize wages in the profile of propor-

\footnotetext{
${ }^{4}$ See Zame (2007) for a firm general equilibrium model with contractual problems.
} 
tions of consumers' types. This parameterization is the ultimate determinant of how the community composition affects agents' utilities. (EGSZ (2006) make no assumptions about the way in which utility depends on the choice of group memberships). This is a fundamental element for our model to capture Berglas' "group composition problem".

\section{Berglas' model reformulated}

Our economy is as follows. Trade of private goods occurs in international markets (as in Berglas (1976, Section 5), where intercommunity trade is allowed for). However, to have access to the consumption of the public goods a consumer must belong to a jurisdiction, and for that the consumer has to acquire a visa permit (or jurisdiction membership). The visa also allows the consumer to work in the jurisdiction industry, and thus to obtain a wage.

The set of private goods is $\mathbf{L}=\{1, \ldots, l, \ldots, L\}$. The corresponding price vector is $p \in \mathbb{R}_{+}^{L}$. There is a finite number of indivisible public projects, in the sense of Mas-Colell (1980). A public project consists of a discrete set of public goods (e.g., schools and parks). The set of public projects is denoted by $\mathbf{G}=\{1, \ldots, g, \ldots, G\}$. There is also a finite set of production technologies $\mathbf{Y}=\{1, \ldots, y, \ldots, Y\}$. The characteristics of these production technologies will be specified below.

In what follows, all of Berglas' (1976) assumptions start with B. Other assumptions needed to assure existence of equilibrium start with A.

Assumption B1: Each public project $g \in \mathbf{G}$ has an associated cost $c(g) \in$ $\mathbb{R}_{+}^{L}$ in terms of private goods.

Assumption B1 says that for each public good (recall that there is a finite number of public goods), there is an associated cost in terms of private goods (and only in terms of private goods). This is a Leontieff type of production technology, where for each type of public good $g \in \mathbf{G}$ the amount of private goods inputs is pre-determined. This assumption is also present in earlier works (see EGSZ (1999) and Konishi (2008)). The next assumption is required in order to avoid non-convexities associated with the consumers' choice problem.

Assumption A1 (Large economy): The set of consumers is a nonatomic finite measure space $(\mathbf{H}, \mathcal{H}, \lambda)$, where $\mathcal{H}$ is a $\sigma$-algebra of subsets of $\mathbf{H}$ and $\lambda$ is the associated Lebesgue measure. 
A consumer's type is a complete description of his endowments, membership characteristics, and preferences (each is described below). The set of consumers' types is finite, denoted by $\boldsymbol{\Theta}=\{1, \ldots, \Theta\}$. Each consumer $h$ in $\mathbf{H}$ has an associated type $\theta \in \boldsymbol{\Theta}$. The set of type $\theta$ consumers is denoted $H(\theta) \in \mathcal{H}$, and has associated a positive finite measure $\lambda(H(\theta))>0$. We refer to a representative consumer of type $\theta \in \boldsymbol{\Theta}$ by $h_{\theta} \in H(\theta)$.

Endowments: The endowment mapping $h_{\theta} \rightarrow e\left(h_{\theta}\right)$ is an integrable function, where $e\left(h_{\theta}\right) \in \mathbb{R}_{+}^{L}$. Endowments are observable. All consumers of the same type are endowed with the same vector of private goods, i.e., $e_{l}\left(h_{\theta}\right)=e_{l}^{\theta}$, for all $l$ and $h_{\theta}$. We assume that endowments are uniformly bounded above and that the aggregate endowment is strictly positive, i.e., $\sum_{\theta} \int_{\mathbf{H}} e\left(h_{\theta}\right) d \lambda>E$, for $E \in \mathbb{R}_{++}^{L}{ }^{5}$

Membership characteristics indicate the role of a consumer type in a jurisdiction. In particular, the consumer's membership characteristic describes his productive skills, that is, his ability to carry out a certain type of work in the jurisdiction industry. Consumers of the same type have the same productive skills. Productive skills are observable and contractible.

Utility function: Consumers have preferences defined over the consumption of private goods $x \in \mathbb{R}_{+}^{L}$, the consumption of the public project $g \in \mathbf{G}$, and its associated level of congestion, denoted by $n \in \mathbb{N}_{+}$(a natural number). Preferences are represented by the utility function $\tilde{u}^{h_{\theta}}(x, g, n)$. This utility is decreasing in the level of congestion. If no public project is offered in a jurisdiction, the congestion is assumed to be irrelevant. ${ }^{6}$ Also, we assume that consumer $h_{\theta}$ 's utility $\tilde{u}^{h_{\theta}}(\cdot, g, n)$ is continuous, strictly monotonic, and quasiconcave. A standard assumption in the club / local public goods literature, which is also present in Berglas (1973), is the following.

Assumption B2: For all $h_{\theta}$ of the same type $\theta, \tilde{u}^{h_{\theta}}(x, g, n)=\tilde{u}^{\theta}(x, g, n)$.

As pointed out in Section 5, Assumption B2 can be relaxed by allowing consumers of the same type to have different preferences on the consumption of private goods.

Jurisdictions and visa permits: A jurisdiction type $\omega$ is described by the membership characteristics of its community and its organizational charac-

\footnotetext{
${ }^{5} \mathrm{By} z \in \mathbb{R}_{++}^{L}$ we mean that all coordinates of the vector $z$ are strictly positive real numbers, whereas by $z \in \mathbb{R}_{+}^{L}$ we mean that all coordinates of the vector $z$ are non-negative real numbers.

${ }^{6}$ That is, $\forall\left(n, n^{\prime}\right)$ with $n \neq n^{\prime}, \tilde{u}^{h_{\theta}}(x, \emptyset, n)=\tilde{u}^{h_{\theta}}\left(x, \emptyset, n^{\prime}\right)$, where $\emptyset$ means that there is no public project.
} 
teristics (in EGSZ (2006) terminology). The organization characteristics of a jurisdiction $\omega$ are its offer of public project, denoted by $g_{\omega} \in \mathbf{G}$, the production technology (described below) associated with the jurisdiction industry, denoted by $y_{\omega} \in \mathbf{Y}$, and the profile of consumers $\left(n_{\omega}^{\theta}\right)_{\theta \in \boldsymbol{\Theta}}$, where $n_{\omega}^{\theta} \in \mathbb{N}$ denotes the number of type $\theta$ consumers in a jurisdiction type $\omega$. The public project has an associated level of congestion $n_{\omega}=\sum_{\theta \in \Theta} n_{\omega}^{\theta}$. Thus, a jurisdiction type is characterized by a policy package $\omega \equiv\left(g_{\omega}, y_{\omega},\left(n_{\omega}^{\theta}\right)_{\theta \in \Theta}\right)$.

For all our analysis, we assume that consumers care only about the level of congestion of the public goods and not about the identities of the other individuals making use of the public goods.

Assumption B3 (Anonymous crowding): For every consumer $h_{\theta} \in \mathbf{H}$ and any pair of jurisdictions $\omega=\left(g_{\omega}, y_{\omega},\left(n_{\omega}^{\theta}\right)_{\theta \in \Theta}\right)$ and $\tilde{\omega}=\left(g_{\tilde{\omega}}, y_{\tilde{\omega}},\left(n_{\tilde{\omega}}^{\theta}\right)_{\theta \in \Theta}\right)$ with $g_{\omega}=g_{\tilde{\omega}}$ and $\sum_{\theta} n_{\omega}^{\theta}=\sum_{\theta} n_{\tilde{\omega}}^{\theta}$, but $n_{\omega}^{\theta} \neq n_{\tilde{\omega}}^{\theta}$ for some consumer types $\theta \in \boldsymbol{\Theta}$, we have $\tilde{u}^{h_{\theta}}\left(x, g_{\omega}, \sum_{\theta} n_{\omega}^{\theta}\right)=\tilde{u}^{h_{\theta}}\left(x, g_{\tilde{\omega}}, \sum_{\theta} n_{\tilde{\omega}}^{\theta}\right)$.

The set of possible jurisdiction types is $\Omega=\{1, \ldots, \omega, \ldots, \Omega\}$. Notice that the set $\Omega$ is finite, since the sets of consumers' types, public projects, and production technologies are finite. Without loss of generality, we refer to a type $\omega$ jurisdiction by $\omega$. The following assumption guarantees that each jurisdiction is negligible with respect to the whole economy.

Assumption A2: Each jurisdiction has a finite number of consumers. ${ }^{7}$

A consequence of imposing assumptions A1 and A2 is that the continuum of consumers splits into finitely populated jurisdictions, ${ }^{8}$ and therefore there will be a continuum of jurisdictions.

By acquiring a visa permit (or membership), a consumer gains access to the jurisdiction, so that he can consume its public goods and work in the jurisdiction industry. The visa is consumer-type $(\theta)$ and jurisdiction-type $(\omega)$ specific. We denote it by $m=(\theta, \omega)$. The set of visas is denoted by $\mathbf{M}$. A list is a function $\iota: \mathbf{M} \rightarrow\{0,1, \ldots\}$ where $\iota(\theta, \omega)$ represents the number of visas of type $(\theta, \omega)$. We

\footnotetext{
${ }^{7}$ The assumption of finitely populated coalitions in an economy with a continuum of consumers is standard in the literature (see Kaneko and Wooders (1986)).

${ }^{8}$ Recently, AW $(2008,2009)$ has dispensed with Assumption A2 by assuming "Desirability of Wealth", so that large political jurisdictions (such as states or countries) become possible in equilibrium. In the present paper we model small communities as entrepreneurial organizations, and thus prefer to keep the idea of macroscopically negligible jurisdictions. Negligible jurisdictions are analogous to Aumann's (1964) pioneering concept of negligible agents.
} 
write Lists $=\{\iota: \iota$ is a list $\}$.

We define $\beta_{\omega}^{\theta}$ as the proportion of type $\theta$ consumers in a jurisdiction type $\omega$, that is, $\beta_{\omega}^{\theta}=\left(n_{\omega}^{\theta} / n_{\omega}\right) \in[0,1]$. We then refer to $\beta_{\omega}=\left(\beta_{\omega}^{\theta}\right)_{\theta \in \Theta}$ as the profile of proportions of consumer types in jurisdiction $\omega$. As explained below, proportions of consumer types are central in our analysis of societal stratification.

Jurisdictions and consumers: In addition to his endowments, membership characteristics, and utility function, a consumer $h_{\theta} \in \mathbf{H}$ has an associated consumption set. We define the consumer $h_{\theta}$ 's consumption set $\mathbf{X}^{h_{\theta}} \subset \mathbb{R}_{+}^{L} \times$ Lists as the set of feasible bundles of private goods and visa permits that consumer $h_{\theta}$ can choose. The consumption set correspondence $h_{\theta} \rightarrow \mathbf{X}^{h_{\theta}}$ is assumed to be a measurable correspondence.

Because consumers choose non-negative numbers of jurisdiction memberships, we define the choice function $\mu: \mathbf{H} \rightarrow$ Lists. For our jurisdictions economy, we impose that $\mathbf{X}^{h_{\theta}}$ is such that:

a) if $\left(x^{h_{\theta}}, \mu^{h_{\theta}}\right) \in \mathbf{X}^{h_{\theta}}$ and $\hat{x}^{h_{\theta}} \geq x^{h_{\theta}}$, then $\left(\hat{x}^{h_{\theta}}, \mu^{h_{\theta}}\right) \in \mathbf{X}^{h_{\theta}}$.

b) each consumer chooses at most one membership, i.e., $\sum_{m \in \mathbf{M}} \mu^{h_{\theta}}(m) \leq 1$.

Condition b) is convenient in order to maintain the idea of a jurisdiction as the place to live and work, as we can examine Berglas' (1976) "group composition problem" in a general equilibrium framework of jurisdictions formation. The set $\operatorname{Lists}\left(h_{\theta}\right)=\left\{\mu^{h_{\theta}} \in \operatorname{Lists}: \sum_{m} \mu^{h_{\theta}}(m) \leq 1, \exists x^{h_{\theta}}\right.$ s.t. $\left.\left(x^{h_{\theta}}, \mu^{h_{\theta}}\right) \in \mathbf{X}^{h_{\theta}}\right\}$ can be seen as the consumer $h_{\theta}$ 's restricted consumption set of visa permits compatible with his private consumption.

The aggregate membership- $(\theta, \omega)$ vector is given by $\hat{\mu}(\theta, \omega)=\int_{H(\theta)} \mu^{h_{\theta}}(\theta, \omega) d \lambda$. We now impose a measurement requirement on $\hat{\mu} \in \mathbb{R}^{\mathbf{M}}$, which requires consistent matching of consumers in terms of the aggregate of choices. ${ }^{9}$ We say that the aggregate membership vector $\hat{\mu} \in \mathbb{R}^{\mathbf{M}}$ is consistent if for every jurisdiction type $\omega \in \boldsymbol{\Omega}$ there is a real number $\gamma(\omega)$ such that $\hat{\mu}(\theta, \omega)=\gamma(\omega) n_{\omega}^{\theta}, \forall \theta \in \mathbf{\Theta}$. Here, $\gamma(\omega)$ is read as the "number" of type $\omega$ jurisdictions. The choice function $\mu: \mathbf{H} \rightarrow$ Lists is consistent for $\tilde{H}=\cup_{\theta} \tilde{H}(\theta) \subseteq \mathbf{H}$ if the corresponding vector $\hat{\mu}$ is consistent. We write Cons $\equiv\left\{\hat{\mu} \in \mathbb{R}^{\mathbf{M}}: \hat{\mu}\right.$ is consistent $\}$. Observe also that under this condition the proportions that hold in a type $\omega$ jurisdiction are maintained once we integrate over the existing jurisdictions of this type, that is, $\frac{\hat{\mu}(\theta, \omega)}{\hat{\mu}(\omega)}=\frac{n_{\omega}^{\theta}}{n_{\omega}}=\beta_{\omega}^{\theta}, \forall \theta \in \boldsymbol{\Theta}$. Also, the relative proportions of consumer types in a

\footnotetext{
${ }^{9}$ See also Kaneko and Wooders (1986) and EGSZ (1999, 2006).
} 
jurisdiction $\omega$ are maintained.

A visa permit $m=(\theta, \omega)$ gives access to the consumption of the public project $g_{\omega} \in \mathbf{G}$ (with an associated level of congestion $n$ ). Thus, we can write consumer $h_{\theta}$ 's utility function as a function of the consumption of private goods and jurisdiction membership, i.e., $u^{h_{\theta}}: \mathbf{X}^{h_{\theta}} \rightarrow \mathbb{R}$. That is, $u^{h_{\theta}}\left(x, \mu^{h_{\theta}}(m)\right) \equiv$ $\tilde{u}^{h_{\theta}}\left(x, g_{\omega}, n_{\omega}\right)$, for $m=\left(\theta, g_{\omega}, y_{\omega},\left(n_{\omega}^{\theta}\right)_{\theta \in \Theta}\right) \cdot{ }^{10}$ The utility mapping $\left(h_{\theta}, x, \mu\right) \rightarrow$ $u^{h_{\theta}}(x, \mu)$ is a jointly measurable function of all its arguments.

Jurisdiction industry: Following Berglas (1976), the production of private goods requires only labor (a body of consumers of different types engaged in the production activity). ${ }^{11}$ In particular, the production correspondence $y \in \mathbf{Y}$ maps labor inputs $\left(\eta^{\theta}\right)_{\theta \in \Theta} \in \mathbb{R}_{+}^{\Theta}$ into private goods (outputs), i.e., $y:\left(\eta^{\theta}\right)_{\theta \in \Theta} \rightrightarrows \mathbb{R}_{+}^{L}$. Individuals with the same productive skills are seen as similar labor units.

Assumption A3: (i) $y\left(\left(\eta^{\theta}\right)_{\theta \in \Theta}\right)$ is convex and compact for all $y \in \mathbf{Y}$, (ii) $\mathbf{0} \in y\left(\left(\eta^{\theta}\right)_{\theta \in \Theta}\right)$ (i.e., no set of consumers is forced to produce), (iii) y is differentiable in labor inputs $\left(\eta^{\theta}\right)_{\theta \in \boldsymbol{\Theta}}$, and (iv) every $y \in \mathbf{Y}$ exhibits a constant returns to scale (CRS) technology. ${ }^{12}$

For each jurisdiction type $\omega$ there is a specific private production technology $y_{\omega} \in \mathbf{Y}$ satisfying Assumption A3.

We consider an economy where the amount of labor contracts available in the jurisdiction industries coincides with the individuals' demand for jurisdictions (in equilibrium). Thus, if in equilibrium a jurisdiction has a profile $\left(n_{\omega}^{\theta}\right)_{\theta \in \Theta}$, then there will be $n_{\omega}^{\theta}$ units of labor of type $\theta$ available in this jurisdiction industry, and therefore, the production of a type $\omega$ jurisdiction evaluated at the profile $\left(n_{\omega}^{\theta}\right)_{\theta \in \Theta}$ will be $y_{\omega}\left(\left(n_{\omega}^{\theta}\right)_{\theta \in \Theta}\right)$.

The wage that a type $\theta$ consumer obtains in a type $\omega$ jurisdiction is denoted by $\alpha_{\omega}^{\theta} \in \mathbb{R}_{+}$. In equilibrium, the consumer's wage equals his marginal productivity in the jurisdiction industry (in our generalized game this is assured by allowing a fictitious auctioneer to mimic the competitive market solution of a production economy). Wages are thus a funciton of prices $p$.

\footnotetext{
${ }^{10}$ The optimization problem with this new form of utility corresponds to Stages 1 and 2 of the generalized game in Section 6 .

${ }^{11} \mathrm{~A}$ model where production is a function of both labor inputs and physical capita would give us no further insights on the group composition problem, and is thus omited.

${ }^{12}$ See Freeman (1996) and Mookherjee and Ray (2010) for the consequences on inequality of considering a different production technology.
} 
Notice that a CRS production technology implies that wages are homogeneous of degree zero in the labor inputs, which in turn implies that the marginal productivity depends on the proportions of consumer types in the jurisdiction. Therefore, in our context, it is legitimate to write the wages as a function of the profile of proportions, i.e., $\alpha_{\omega}^{\theta}\left(p, \beta_{\omega}\right), \forall \theta \in \boldsymbol{\Theta}$. This parameterization is the final justification of assuming a finite number of consumer types, as the community composition can be easily described in terms of proportions.

Pricing: Consumers must pay personalized lump-sum taxes in order to cover the cost of providing the public project $g_{\omega}$ offered by a jurisdiction $\omega$. In particular, a type $\theta$ consumer will pay a transfer $t_{\omega}^{\theta}$ in jurisdiction $\omega$ and a poll fee $p \cdot \tau_{\omega}$, with $\tau_{\omega} \in \mathbb{R}_{+}^{L}$. The poll fees, common to all consumers in the jurisdiction, cover the costs $p c\left(g_{\omega}\right)$ of providing the public project $g_{\omega}$. The transfers $t_{\omega} \in \mathbb{R}^{\Theta}$ can be positive, negative, or zero, as transfers internalize the externalities among the consumers in the jurisdiction, given their tastes for the public goods. Consumers' types are observable and thus all consumers of the same type $\theta$ in the same jurisdiction type $\omega$ pay the same transfer $t_{\omega}^{\theta}$. We say that $t_{\omega} \in \mathbb{R}^{\Theta}$ is a pure transfer system in jurisdiction $\omega$ if $t_{\omega} \in$ Trans, where Trans $=\left\{t_{\omega} \in \mathbb{R}^{\Theta}: \sum_{\theta} t_{\omega}^{\theta} \hat{\mu}(\theta, \omega)=0, \forall \hat{\mu} \in\right.$ Cons $\}$.

The consumer's budget constraint associated with his choice of jurisdiction membership $\mu^{h_{\theta}}(\theta, \omega)$ is

$$
p x^{h_{\theta}}+t_{\omega}^{\theta}+p \tau_{\omega} \leq \alpha_{\omega}^{\theta}+p e^{\theta} \quad\left(\mathrm{BC}_{h_{\theta}}(\omega)\right)
$$

The budget constraint $\mathrm{BC}_{h_{\theta}}(\omega)$ says that the sum of the cost of the commodities purchased $p x^{h_{\theta}}$ and the tax $t_{\omega}^{\theta}+p \tau_{\omega}$ required to finance the cost of the public goods of the jurisdiction must be less than or equal to the type-and-jurisdictionspecific-wage $\alpha_{\omega}^{\theta}$ that the consumer obtains by working in the jurisdiction industry, and the income obtained from selling the consumer's endowments of private goods.

Consumers of a given type can be required to satisfy some capital provision in order to acquire a certain jurisdiction membership. Let us think of this commodity as land and denote it by $\tilde{l} \in \mathbf{L}$. Land can be acquired in the market by any agent, but only those consumers with this land requirement have the obligation to accommodate it in their consumption bundles. That is, for a consumer $h_{\tilde{\theta}}$, with $\left(x^{h_{\tilde{\theta}}}, \mu^{h_{\tilde{\theta}}}(\tilde{\theta}, \omega)\right) \in \mathbf{X}^{h_{\tilde{\theta}}}$, if $\mu^{h_{\tilde{\theta}}}(\tilde{\theta}, \omega)=1$ for a jurisdiction membership $m=(\tilde{\theta}, \omega)$ that requires providing $k$ units of land, then $x_{\tilde{l}}^{h_{\tilde{\theta}}} \geq k$.

Let $U^{h_{\theta}}\left(\omega ; p, t_{\omega}^{\theta}, \tau_{\omega}, \alpha_{\omega}^{\theta}\right)=\left\{\max _{\{x\}} u^{h_{\theta}}\left(x, \mu^{h_{\theta}}(\theta, \omega)\right): \mathrm{BC}_{h_{\theta}}(\omega)\right.$ holds $\}$ be the 
indirect utility of a consumer $h_{\theta}$ with a membership in a jurisdiction type $\omega$, given the prices $p, t_{\omega}^{\theta}, \tau_{\omega}$, and $\alpha_{\omega}^{\theta}$.

Equilibrium: We consider the notion of a price-taking equilibrium (prices precede maximization) as an efficient summary of the equilibrium corresponding to a competitive theory of jurisdictions.

Definition 1: An equilibrium for this Berglas economy consists of a vector of bundles and memberships $(x, \mu)$ and prices $(p, t, \tau, \alpha)$ such that:

(E.1) Consumers choose optimally: if there exists $\left(\tilde{x}_{\tilde{\omega}}^{h_{\theta}}, \tilde{\mu}^{h_{\theta}}(\theta, \tilde{\omega})\right) \in \mathbf{X}^{h_{\theta}}$ such that $u^{h_{\theta}}\left(\tilde{x}_{\tilde{\omega}}^{h_{\theta}}, \tilde{\mu}^{h_{\theta}}(\theta, \tilde{\omega})\right)>u^{h_{\theta}}\left(x_{\omega}^{h_{\theta}}, \mu^{h_{\theta}}(\theta, \omega)\right)$, then the membership $\tilde{\mu}^{h_{\theta}}(\theta, \tilde{\omega})$ is such that $p\left(\tilde{x}_{\tilde{\omega}}^{h_{\theta}}-e^{\theta}\right)+t_{\tilde{\omega}}^{\theta}+p \tau_{\tilde{\omega}}-\alpha_{\tilde{\omega}}^{\theta}>0$.

(E.2) No profits in the provision of public goods:

$\sum_{\theta \in \boldsymbol{\Theta}} n_{\omega}^{\theta}\left(t_{\omega}^{\theta}+p \tau_{\omega}\right)=p c\left(g_{\omega}\right), \forall \omega \in \mathbf{\Omega}$.

(E.3) No profits in the production of private goods:

$p y_{\omega}\left(\left(n_{\omega}^{\theta}\right)_{\theta \in \boldsymbol{\Theta}}\right)=\sum_{\theta} \alpha_{\omega}^{\theta} n_{\omega}^{\theta}, \forall \omega \in \boldsymbol{\Omega}$.

(E.4) Market clearing for private goods:

$\sum_{\theta} \int_{H(\theta)}\left(x^{h_{\theta}}-e^{\theta}\right) d \lambda+\sum_{\omega} \gamma(\omega)\left(c\left(g_{\omega}\right)-y_{\omega}\left(\left(n_{\omega}^{\theta}\right)_{\theta \in \boldsymbol{\Theta}}\right)\right)=0$.

(E.5) Consistency: $\hat{\mu}$ is consistent for $\mathbf{H}=\cup_{\theta} H(\theta)$.

The following assumption ("Desirability of endowments") is needed in order to assure that the weak core and the strong core coincide (we refer to EGSZ (1999) for the distinction between both concepts). Our Assumption A4 differs slightly from EGSZ (2006) in that here we address the possibility that some visas may require some consumers to satisfy some minimum consumption of capital.

Assumption A4 (Endowments are desirable): For every consumer $h_{\theta} \in$ $\mathbf{H}$ and every consumption choice $\left(x^{h_{\theta}}, \mu^{h_{\theta}}\right) \in \mathbf{X}^{h_{\theta}}$ for which $u^{h_{\theta}}\left(x^{h_{\theta}}, \mu^{h_{\theta}}\right)>$ $u^{h_{\theta}}\left(e^{h_{\theta}}, 0\right)$, there exists $\tilde{x}^{h_{\theta}} \leq x^{h_{\theta}}, \tilde{x}^{h_{\theta}} \neq x^{h_{\theta}}$, such that $\left(\tilde{x}^{h_{\theta}}, \mu^{h_{\theta}}\right) \in \mathbf{X}^{h_{\theta}}$ and $\tilde{x}^{h_{\theta}} \geq k$, where $k \geq 0$ stands for the capital requirement of membership $\mu^{h_{\theta}}$.

The last remaining assumption (A5) is needed in order to prevent the "minimum expenditure situation" (see EGSZ (1999) for an example where a quasiequilibrium fails to be an equilibrium in an economy where the private goods are used as inputs for the production of public projects). Let us first introduce the following definitions. The pair $(x, \mu)$ is said to be a feasible state for a measurable set $H \subseteq \mathbf{H}$ if $(x, \mu) \in \mathbf{X}^{h_{\theta}}$ satisfies the budget constraints for each $h_{\theta} \in H$ 
and, for the set of consumers $H$, conditions (E.4) and (E.5) hold. Now let $\delta_{l^{\prime}}$ be a vector in $\mathbb{R}_{+}^{L}$ consisting of one unit of the $l^{\prime}$ commodity and nothing else. Say that $(x, \mu)$ is jurisdiction linked if whenever $\hat{L} \cup \bar{L}=\mathbf{L}$ is a partition of the set of private goods and $x_{l}^{h}=0$ for all $l \in \hat{L}$ and almost all consumers $h \in \mathbf{H}$, then for almost all consumers $h_{\theta} \in \mathbf{H}$ there exists $r>0$ and $l^{\prime} \in \bar{L}$ such that $u^{h_{\theta}}\left(e^{\theta}+r \delta_{l^{\prime}}, 0\right)>u^{h_{\theta}}\left(x^{h_{\theta}}, \mu^{h_{\theta}}(\theta, \omega)\right), \forall \theta .{ }^{13}$ We say that our economy is jurisdiction irreducible if every feasible allocation is jurisdiction linked.

Assumption A5: Our economy is jurisdiction irreducible.

\section{Results}

Theorem 1 (Existence): Let assumptions A1-A5 and B1-B3 hold for this Berglas economy. Then, there exists an equilibrium.

In Section 5 we explain the novel aspects of our simultaneous optimization approach for proving Theorem 1 and the main differences with earlier proofs (core decentralization approach (see Conley and Wooders (1997) and ACW (2009)) and non-excess demand approach (see EGSZ (1999)). In Section 6 we present the generalized game and the proof.

In our next result we establish the core equivalence theorem for our economy. Let us first introduce the following definitions. We say that $(x, \mu)$ is in the core if there is no subset of $H \subset \mathbf{H}$ with $\lambda(H)>0$ and a feasible state $(\tilde{x}, \tilde{\mu})$ for $H$ such that $u^{h_{\theta}}\left(\tilde{x}^{h_{\theta}}, \tilde{\mu}^{h_{\theta}}\right) \geq u^{h_{\theta}}\left(x^{h_{\theta}}, \mu^{h_{\theta}}\right)$ for all types $\theta \in \boldsymbol{\Theta}$ and all $h_{\theta} \in H$, and $u^{h_{\theta}^{\prime}}\left(\tilde{x}^{h_{\theta}^{\prime}}, \tilde{\mu}^{h_{\theta}^{\prime}}\right)>u^{h_{\theta}^{\prime}}\left(x^{h_{\theta}^{\prime}}, \mu^{h_{\theta}^{\prime}}\right)$ for all types $\theta \in \Theta$ and all $h_{\theta}^{\prime} \in H^{\prime} \subset H$ with $\lambda\left(H^{\prime}\right)>0$. A feasible state $(x, \mu)$ is Pareto optimal if there is no feasible state $(\tilde{x}, \tilde{\mu})$ for $\mathbf{H}$ such that $u^{h_{\theta}}\left(\tilde{x}^{h_{\theta}}, \tilde{\mu}^{h_{\theta}}\right) \geq u^{h_{\theta}}\left(x^{h_{\theta}}, \mu^{h_{\theta}}\right)$ for almost all $h_{\theta} \in \mathbf{H}$ and $u^{h_{\theta}^{\prime}}\left(\tilde{x}^{h_{\theta}^{\prime}}, \tilde{\mu}^{h_{\theta}^{\prime}}\right)>u^{h_{\theta}^{\prime}}\left(x^{h_{\theta}^{\prime}}, \mu^{h_{\theta}^{\prime}}\right)$ for all $h_{\theta}^{\prime} \in H^{\prime} \subset \mathbf{H}$ with $\lambda\left(H^{\prime}\right)>0$.

The following assumption is needed in order to guarantee that every core state can be supported as an equilibrium. It requires that, for every jurisdiction type $\omega=\left(g_{\omega}, y_{\omega},\left(n_{\omega}^{\theta}\right)_{\theta \in \boldsymbol{\Theta}}\right)$, (i) the profile of consumers $\left(n_{\omega}^{\theta}\right)_{\theta \in \boldsymbol{\Theta}}$ satisfies the optimal

\footnotetext{
${ }^{13}$ This is read as follows: if the entire social endowment of private goods is used to produce public projects, then, for almost every consumer $h_{\theta} \in \mathbf{H}$ and every $\theta \in \mathbf{\Theta}$, there exists some good $l^{\prime} \notin \hat{L}$ and some sufficiently large level of consumption of this good such that every agent would prefer to consume his endowment together with this large level of good $l^{\prime}$, and belong to no jurisdiction, rather than to consume the bundle $x^{h_{\theta}}$ in the jurisdiction membership $\mu^{h_{\theta}}(\theta, \omega)$.
} 
proportions $\beta_{\omega}$ that maximize the CRS production technology $y_{\omega}$, and (ii) the positive measure $\gamma(\omega)$ is absolutely continuous with respect to the measure $\lambda$. For the latter assumption let us first define the set of consumers with membership $m=(\theta, \omega)$ by $\hat{\mathbf{H}}_{\omega}=\left\{h \in H: \mu^{h_{\theta}}(\theta, \omega)=1, \forall \theta\right\}$, and denote by $\hat{H}_{\omega}$ a subset of $\hat{\mathbf{H}}_{\omega}$ for which $\hat{\mu}$ is consistent, i.e., $\hat{H}_{\omega}=\left\{h \in \hat{\mathbf{H}}_{\omega}: \exists \gamma(\omega)\right.$ s.t. $\hat{\mu}(\theta, \omega)=\gamma(\omega) n_{\omega}^{\theta}$, $\forall \theta\}$. Recall that if a state $(x, \mu)$ is in the core, then $\mu$ is consistent by definition, and therefore, such a measure $\gamma$ exists. Denote the measure space associated with $\hat{\mathbf{H}}_{\omega}$ by $\left(\hat{\mathbf{H}}_{\omega}, \mathcal{H}_{\omega}, \lambda\right)$, where $\mathcal{H}_{\omega}$ is the set of subsets $\hat{H}_{\omega}$ of $\hat{\mathbf{H}}_{\omega}$. The production associated with $\hat{H}_{\omega} \in \mathcal{H}_{\omega}$ is then $y_{\omega}\left(\hat{H}_{\omega}\right)=\gamma(\omega) y_{\omega}\left(\left(n_{\omega}^{\theta}\right)_{\theta \in \boldsymbol{\Theta}}\right)$.

Assumption A6: For every $\omega \in \boldsymbol{\Omega}$, (i) $n_{\omega}^{\theta} / n_{\omega}=\beta_{\omega}^{\theta}$ for all $\theta \in \boldsymbol{\Theta}$, where $\beta_{\omega}$ are the proportions that maximize the CRS production technology $y_{\omega}$, and (ii) if $\lambda\left(H_{\omega}\right)=0$, then $y_{\omega}\left(\hat{H}_{\omega}\right)=0$.

Theorem 2 (Core equivalence): Let Assumptions A1-A4 and B1-B3 hold for this Berglas economy. Then, every equilibrium is in the core. If also Assumptions $A 5$ and $A 6$ are imposed, then the core coincides with the set of equilibria.

We leave the proof of Theorem 2 for Section 6. We incorporate to the proof of EGSZ (1999, Theorem 5.1) the private goods production side. For that, we must accommodate the total production of private goods in order to find prices $(p, t) \in \mathbb{R}^{L} \times \mathbb{R}^{|\mathbf{M}|},(p, t) \neq(0,0)$, that separate the two constructed convex cones. But our main difficulty is to show that the there exists a measurable function $\alpha_{\omega}\left(p, \beta_{\omega}, \cdot\right)$ of $\mathcal{H}_{\omega}$ into $\mathbb{R}_{+}$such that, for every $\hat{H}_{\omega} \in \mathcal{H}_{\omega}, p y_{\omega}\left(\hat{H}_{\omega}\right)=$ $\int_{\hat{H}_{\omega}} \alpha_{\omega}\left(p, \beta_{\omega}, \cdot\right) d \lambda$, for any given $p$. What is important to see here is that without Assumption A6 the mapping $p y_{\omega}(\cdot)$ of $\mathcal{H}_{\omega}$ into $\mathbb{R}_{+}$fails to be countably additive and, therefore, the existence of a Radon-Nykodyn derivative $\alpha_{\omega}^{\theta}\left(p, \beta_{\omega}, \cdot\right)$ is not guaranteed (see Hildebrand (1968)). Countable additivity of the mapping $p y_{\omega}(\cdot)$ fails if, for a countable family $\left\{H_{\omega}^{i}\right\}_{i \in \mathbf{I}}$ of pairwise disjoint subsets of $\hat{\mathbf{H}}_{\omega}$, it happens that $p y_{\omega}\left(\cup_{i} H_{\omega}^{i}\right) \neq \sum_{i} p y_{\omega}\left(H_{\omega}^{i}\right)$. A constant returns to scale technology $y_{\omega}$ is maximized only at the optimal proportions $\beta_{\omega}$. Unfortunately, there might exist sets $\left(H_{\omega}^{i}\right)_{i \in \mathbf{I}} \in \mathcal{H}_{\omega}$ and a consistent choice function where the union $\cup_{i} H_{\omega}^{i}$ exhibits the optimal proportions, but where the proportions of consumers' types for each of these sets $\left(H_{\omega}^{i}\right)_{i \in \mathbf{I}}$ are not optimal. 


\section{Heterogeneous Tiebout communities}

Indirect utility and proportions: We write the consumer $h_{\theta}$ 's indirect utility with a membership $m=(\theta, \omega)$ as a function of the proportions $\beta_{\omega}=\left(\beta_{\omega}^{\theta}\right)_{\theta \in \Theta}$ and the level of congestion $n_{\omega}$, that is, $U^{h_{\theta}}\left(g_{\omega}, y_{\omega}, n_{\omega}, \beta_{\omega} ; p, t_{\omega}^{\theta}, \tau_{\omega}, \alpha_{\omega}^{\theta}\right)$. Observe that $U^{h_{\theta}}\left(g_{\omega}, y_{\omega},\left(n_{\omega}^{\theta}\right)_{\theta \in \boldsymbol{\Theta}} ; p, t_{\omega}^{\theta}, \tau_{\omega}, \alpha_{\omega}^{\theta}\right)$ can be rewritten as above using two arguments, $\beta_{\omega}$ and $n_{\omega}$ (by letting $n_{\omega}^{\theta}=\beta_{\omega}^{\theta} n_{\omega}$ ). The first argument (the proportions $\beta_{\omega}$ ) enter into the consumer's budget constraint through the wages $\alpha_{\omega}^{\theta}\left(p, \beta_{\omega}\right)$ due to our Assumption A3 (CRS production technology), and therefore the proportions $\beta_{\omega}$ can be used as a direct argument of $U^{h_{\theta}}$. The second argument (the level of congestion $n_{\omega}$ ) enter as a direct argument of $U^{h_{\theta}}$, since the primitive utility function $\tilde{u}^{h_{\theta}}$ depends on $n_{\omega}$ (by Assumption B3). Notice that the identities of the other consumers making use of the jurisdiction public goods do not appear in $U^{h_{\theta}}\left(g_{\omega}, y_{\omega}, n_{\omega}, \beta_{\omega} ; p, t_{\omega}^{\theta}, \tau_{\omega}, \alpha_{\omega}^{\theta}\right)$, consistent with our assumption B3 (anonymous crowding).

Index of heterogeneity: Let us now propose the following index of heterogeneity $(\mathrm{H})$ in a jurisdiction:

$$
I_{\omega}\left(\theta, \theta^{\prime}\right)=\left|\max _{\theta} \beta_{\omega}^{\theta}-\min _{\theta^{\prime}} \beta_{\omega}^{\theta^{\prime}}\right| \in[0,1]
$$

The heterogeneity in a jurisdiction can be classified as follows. We say that a community is mixed (or heterogeneous) when there is at least more than one consumer type in it, that is, there exists $\left(\theta, \theta^{\prime}\right)$ with $\theta \neq \theta^{\prime}$ such that $I_{\omega}\left(\theta, \theta^{\prime}\right) \neq 1$. We denote the profile of proportions of a heterogeneous community $\omega_{H E T}$ by $\beta_{\omega_{H E T}}$. We shall say that two jurisdictions $\omega$ and $\omega^{\prime}$ have the same vector of proportions if for all $\theta \in \boldsymbol{\Theta}, \beta_{\omega}^{\theta}=\beta_{\omega^{\prime}}^{\theta}$, that is, if $I_{\omega}\left(\theta, \theta^{\prime}\right)=0, \forall\left(\theta, \theta^{\prime}\right)$. When $I_{\omega}\left(\theta, \theta^{\prime}\right)=0$ and $\min \left\{\beta_{\omega}^{\theta}, \beta_{\omega}^{\theta^{\prime}}\right\} \neq 0$, the two types $\theta$ and $\theta^{\prime}$ of consumers are in the same proportions in a jurisdiction type $\omega$, and therefore, the jurisdiction type $\omega$ is uniformly heterogeneous on these two types of consumers. When $I_{\omega}\left(\theta, \theta^{\prime}\right)>0$ and $\min \left\{\beta_{\omega}^{\theta}, \beta_{\omega}^{\theta^{\prime}}\right\} \neq 0$, the jurisdiction $\omega$ is mix-populated by the two types of consumers but in different proportions. If $I_{\omega}\left(\theta, \theta^{\prime}\right)=1, \forall \theta^{\prime} \neq \theta$, then the jurisdiction type has a homogeneous population of type $\theta$ consumers, that is, $\beta_{\omega_{H O M(\theta)}}$ has a vector $(0, \ldots, 1, \ldots, 0)$ with 1 in the $\theta$ component but 0 otherwise.

Let us now provide a sufficient (endogenous) condition for the existence of heterogeneous Tiebout communities, useful for performing comparative statics among the set of equilibria.

Equilibrium comparative statics: An (endogenous) sufficient condition 
for the formation of a heterogeneous community is that, for every consumer $h_{\theta}$ and every type of homogeneous jurisdiction $\omega_{H O M(\theta)}$,

$U^{h_{\theta}}\left(\omega_{H E T} ; p, t_{\omega_{H E T}}^{\theta}, \tau_{\omega_{H E T}}, \alpha_{\omega_{H E T}}^{\theta}\right)>U^{h_{\theta}}\left(\omega_{H O M(\theta)} ; p, t_{\omega_{H O M(\theta)}}^{\theta}, \tau_{\omega_{H O M(\theta)}}, \alpha_{\omega_{H O M(\theta)}}^{\theta}\right)$

(ESC1)

This condition is endogenous to the model, as it depends on equilibrium variables, such as equilibrium prices. A more intuitive (endogenous) condition could be obtained if one considered a separable utility function ${ }^{14}$ of the form $\tilde{u}^{h_{\theta}} \equiv \tilde{u}^{h_{\theta}}\left(x_{\omega}\right)+\tilde{u}^{h_{\theta}}\left(g_{\omega}, n_{\omega}\right)$. Then, an (endogenous) sufficient condition for the formation of a heterogeneous community $\omega_{H E T}$ is that, for every consumer $h_{\theta}$ and every type of homogeneous jurisdiction $\omega_{H O M(\theta)}$,

$$
\tilde{u}^{h_{\theta}}\left(\bar{x}_{\omega_{H E T}}^{h_{\theta}}\right)+\tilde{u}^{h_{\theta}}\left(g_{\omega_{H E T}}, n_{\omega_{H E T}}\right)>\tilde{u}^{h_{\theta}}\left(\bar{x}_{\omega_{H O M}(\theta)}^{h_{\theta}}\right)+\tilde{u}^{h_{\theta}}\left(g_{\omega_{H O M}(\theta)}, n_{\omega_{H O M(\theta)}}\right)
$$

where $\bar{x}^{h_{\theta}} \in \arg \max u^{h_{\theta}}\left(x, \mu^{h_{\theta}}(\theta, \omega).\right)$ such that $\mathrm{BC}_{h_{\theta}}(\omega)$ holds, given the pair $\left(y_{\omega}, \beta_{\omega}\right)$. (ESC2) guarantees that the loss in utility from not consuming the most preferred public goods in $\omega_{H E T}\left(\tilde{u}^{h_{\theta}}\left(g_{\omega_{H E T}}, n_{\omega_{H E T}}\right)<\tilde{u}^{h_{\theta}}\left(g_{\omega_{H O M(\theta)}}, n_{\omega_{H O M(\theta)}}\right)\right)$ is more than compensated by the higher wages that the consumer obtains in the heterogeneous community, in turn increasing consumer's utility through a greater consumption of private goods, due to the good labor complementarities and high productivity of the jurisdiction industry. Glaeser and Saiz (2004) provide an empirical justification of this effect. These authors show that skilled cities are growing because they are becoming more economically productive, not because these cities are becoming more attractive places to live.

Necessary and sufficient conditions on the primitives: In a Berglas (1976) economy, where the production of private goods depends on labor inputs and consumers do not care about the identities of the other consumers that consume the jurisdiction public goods, it is easy to see that there are two necessary conditions on the primitives for the existence of a mixed community, already identified by Berglas (1976).

(NC1) Labor skills must be different among consumers.

Condition (NC1) is necessary. To see this, we can think of an economy with

\footnotetext{
${ }^{14}$ Assumption A5, which guarantees that private goods are essential (Mas-Colell (1980)), excludes separability between private and local public goods. Guilles and Scotchmer (1997) show that the "essentiality of private goods" assumption can be dispensed with if one considers instead the assumptions "exhaustion of blocking opportunities" and "efficient scale".
} 
anonymous crowding where all types of consumers have identical labor skills but different preferences on public goods. These consumers cannot increase their utility by producing in an industry with a production technology based on labor complementarities, and therefore the consumers will be better off in the standard homogeneous Tiebout communities.

(NC2) Labor complementarities in the production of private goods, i.e., there exists a production technology $y \in \mathbf{Y}$ such that $\frac{d\left(y\left(\left(\eta^{\theta}\right)_{\theta \in \Theta}\right)\right)}{d \eta^{1} \cdot \ldots \cdot \eta^{\Theta}}>0$.

In the absence of a collaborative production process, the well known result that homogenous groups coalesce around like individuals will apply in an economy with anonymous crowding. See Scotchmer and Wooders (1987) for this result and McGuire (1991) for a detailed normative analysis on this issue.

We finish with a sufficient condition on the primitives that assures that, if there are two types of consumers moving from a homogeneous jurisdiction to an heterogeneous jurisdiction that experience sufficiently rich increments in their marginal productivities (due to the complementarities in the joint production process), then mixed communities will form in equilibrium. In Proposition 1 we restrict to the simplest scenario of a one good production economy, although there can be several goods transacted in this economy. This assumption is needed in order to associate the consumer's wage with his marginal productivity in production, but where prices are absent (since the price of the produced good can be normalized to one).

Proposition 1 (Sufficient condition): Assume that Assumptions A1-A6 and B1-B3 hold. Also assume that all jurisdiction industries produce only one good. Let $\bar{\omega}=\left(g_{\bar{\omega}}, y_{\bar{\omega}},\left(n_{\bar{\omega}}^{\theta}, n_{\bar{\omega}}^{\theta^{\prime}}\right)\right)$ be a jurisdiction type with $y_{\bar{\omega}}$ satisfying (NCZ). Then, there exists an equilibrium with heterogenous communities if there is a group of consumers $H \in \mathcal{H}$ formed only by consumer types $\theta$ and $\theta^{\prime}$ satisfying (NC1), and a vector $\varepsilon=(\varepsilon, \ldots, \varepsilon) \in \mathbb{R}_{++}^{L}$ such that, for any pair of bundles $x, x^{\prime} \in$ $\mathbb{R}_{+}^{L}$ and pair of jurisdiction types $\left(\omega, \omega^{\prime}\right)=\left(\left(g_{\omega}, y_{\omega}, n_{\omega}^{\theta}\right),\left(g_{\omega^{\prime}}, y_{\omega^{\prime}}, n_{\omega^{\prime}}^{\theta^{\prime}}\right)\right)$, we have that $\tilde{u}^{h_{\theta}}\left(x+\varepsilon, g_{\bar{\omega}}, n_{\bar{\omega}}\right)>\tilde{u}^{h_{\theta}}\left(x, g_{\omega}, n_{\omega}\right)$ and $\tilde{u}^{h_{\theta^{\prime}}}\left(x^{\prime}+\varepsilon, g_{\bar{\omega}}, n_{\bar{\omega}}\right)>\tilde{u}^{h_{\theta^{\prime}}}\left(x^{\prime}, g_{\omega^{\prime}}, n_{\omega^{\prime}}\right)$ for all $h_{\theta}, h_{\theta^{\prime}} \in H$, and such that the weighted productivity increment

$$
\Delta \equiv \beta_{\bar{\omega}}^{\theta^{\prime}}\left(\frac{\partial y_{\bar{\omega}}\left(n_{\bar{\omega}}^{\theta}, n_{\bar{\omega}}^{\theta^{\prime}}\right)}{\partial \eta^{\theta^{\prime}}}-\frac{\partial y_{\omega^{\prime}}\left(n_{\omega^{\prime}}^{\theta^{\prime}}\right)}{\partial \eta^{\theta^{\prime}}}\right)+\beta_{\bar{\omega}}^{\theta}\left(\frac{\partial y_{\bar{\omega}}\left(n_{\bar{\omega}}^{\theta}, n_{\bar{\omega}}^{\theta^{\prime}}\right)}{\partial \eta^{\theta}}-\frac{\partial y_{\omega}\left(n_{\omega}^{\theta}\right)}{\partial \eta^{\theta}}\right)
$$

satisfies

$$
\Delta>\varepsilon+\max _{(\tilde{\theta}, \tilde{\omega})=(\theta, \omega),\left(\theta^{\prime}, \omega^{\prime}\right)} \beta_{\bar{\omega}}^{\tilde{\theta}} \frac{\partial y_{\tilde{\omega}}\left(n_{\tilde{\omega}}^{\tilde{\theta}}\right)}{\partial \eta^{\tilde{\theta}}}+2 \max \left\{\beta_{\bar{\omega}}^{\theta^{\prime}}, \beta_{\bar{\omega}}^{\theta}\right\} \max _{l, \bar{\theta}} e_{l}^{\bar{\theta}}+\max _{l} \frac{c_{l}\left(g_{\bar{\omega}}\right)}{n_{\bar{\omega}}}
$$


Condition (SC) says that the weighted increase in productivity from a state composed by homogeneous jurisdictions $\left(\omega\right.$ and $\left.\omega^{\prime}\right)$ to another state composed by an heterogeneous jurisdiction $(\bar{\omega})$ must be enough to pay for: 1) the consumption increment $\varepsilon>0,2)$ the highest productivity between the two types of consumers when producing in homogeneous communities, weighted by the proportions of this type of consumer in the heterogeneous community $\bar{\omega}, 3)$ the double of the highest individual good endowment weighted by the highest proportion of consumer types in $\bar{\omega}$, and 4 ) the highest possible per capita cost in terms of one good required to constitute the jurisdiction $\bar{\omega}$.

The intuition for Proposition 1 is that mixed communities are optimal and exist in equilibrium if there exists two types of consumers with a consumption increment $\varepsilon>0$ satisfying (SC), and for whose gains from labor complementarities (through higher wages) outweigh the cost (in utility terms) of not consuming the most preferred public goods in the jurisdiction. Observe that the sufficient condition in Proposition 1 is obtained only on the primitives of our economy, and that the pair $\left(x, x^{\prime}\right)$ can be any pair of consumption bundles, not necessarily the equilibrium ones. Also notice that Proposition 1 does not assert that the heterogeneous communities that form in equilibrium will be of type $\bar{\omega}$, neither says which will be the equilibrium consumption bundle.

The following examples demonstrates that condition (SC) is not necessary for the existence of optimal heterogeneous communities; it is only a sufficient condition. Also, the example illustrates the group composition problem that arises when wages depend on a profile of different types of labor and consumers have different tastes for public goods.

Example 1: Let there be two types of consumers, $\theta=1,2$, with the same measure for each type, i.e., $\lambda^{1}=\lambda^{2} \equiv \lambda$. There is only one perfectly divisible good, the price of which is 1 . Good endowments are $e^{1}=3$ and $e^{2}=2$, respectively. Let us consider three types of jurisdiction, $\omega_{1}=\left(g_{1}, y_{S},(2,0)\right)$, $\omega_{2}=\left(g_{2}, y_{S},(0,2)\right)$ and $\omega_{3}=\left(g_{3}, y_{C},(1,1)\right)$. For tractability, we assume $n_{\omega}=2$, for all $\omega=\omega_{1}, \omega_{2}, \omega_{3}$, so congestion is not a decisive factor to discriminate among jurisdiction types. The production technology $y_{C}$ is collaborative, with the following form: $y_{C}=20 \sqrt{n^{1} n^{2}}$. The labor inputs of the production technology $y_{S}$ are perfect substitutes, with functional form $y_{S}=3 n^{1}+n^{2}$. The equilibrium wages are $\alpha_{\omega_{1}}^{1}=3, \alpha_{\omega_{2}}^{2}=1$ and $\alpha_{\omega_{3}}^{1}=\alpha_{\omega_{3}}^{2}=10$. The three public goods cost the same, $c\left(g_{\omega}\right)=2, \omega=\omega_{1}, \omega_{2}, \omega_{3}$, although all differ in terms of their characteris- 
tics (say, in their location). Utilities are defined as follows: $\tilde{u}^{1}\left(x, g_{1}, 2\right)=\frac{1}{3} \sqrt{x}$, $\tilde{u}^{1}\left(x, g_{3}, 2\right)=\frac{1}{4} \sqrt{x}, \tilde{u}^{2}\left(x, g_{2}, 2\right)=\frac{1}{4} \sqrt{x}$ and $\tilde{u}^{2}\left(x, g_{3}, 2\right)=\frac{1}{5} \sqrt{x}$.

For these parameters there is no $\varepsilon>0$ that satisfies (SC). However, heterogeneous communities of type $\omega_{3}$ will form in equilibrium. ${ }^{15}$ Prices are $p=1, \tau_{\omega}=1$ for all $\omega=\omega_{1}, \omega_{2}, \omega_{3}, t_{\omega_{1}}^{1}=t_{\omega_{2}}^{2}=0$ (since jurisdictions $\omega_{1}$ and $\omega_{2}$ are composed of homogeneous consumers) and $t_{\omega_{3}}^{1}=-t_{\omega_{3}}^{2}=-2.02 .{ }^{16}$ Indirect utilities are $U^{1}\left(\omega_{1} ; 1,0,1,3\right)=0.74, U^{1}\left(\omega_{3} ; 1,-2.02,1,10\right)=0.93, U^{2}\left(\omega_{2} ; 1,0,1,1\right)=0.35$, and $U^{2}\left(\omega_{3} ; 1,2.02,1,3\right)=0.59$. Both types of consumers are better off in jurisdiction $\omega_{3}$, even if both consumer types consume the less preferred public good $\left(g_{3}\right)$. This is because wages are higher in $\omega_{3}$ than in both $\omega_{1}$ and $\omega_{2}$ due to the labor complementarities in the production technology $y_{C}$. Thus, we can conclude that the core is characterized by heterogeneous communities with profile $\left(n^{1}, n^{2}\right)=(1,1)$. Consistency holds by making $\gamma\left(\omega_{3}\right)=\lambda$. Assumption A6 holds for our restricted set of jurisdiction types. Thus, by Theorem 2, we know that the core coincides with the set of equilibria. Therefore, we can claim that, for this example, the Berglas equilibrium is characterized by heterogeneous Tiebout communities of type $\omega_{3}=\left(g_{3}, y_{C},(1,1)\right)$.

\section{Final remarks}

Visa permits and pricing: Each member evaluates a visa $m=(\theta, \omega)$ through the corresponding personalized membership price $q_{\omega}^{\theta} \equiv t_{\omega}^{\theta}+p \tau_{\omega}-\alpha_{\omega}^{\theta}$ (tax net of wage). The pricing of visas in our framework is more complex than it would be in a standard local public good economy, as the tax system depends on the community composition, but also the wages depend on the labor complementarities of the workers in the jurisdiction industry. Observe that it may occur that, even if $t_{\omega}^{\theta}+p \tau_{\omega}>0$, we have $q_{\omega}^{\theta}<0$ (negative visa price), as the wage more than compensates the tax payment. In this sense, the wage $\alpha_{\omega}^{\theta}$ can be seen as a lump-sum premium (or subsidy) paid to the consumer for his good (bad) labor complementaries with the other consumers of his jurisdiction. Below, in our generalized game (Section 6), the personalized membership price $q_{\omega}^{\theta}$ is decomposed

\footnotetext{
${ }^{15}$ If endowments were $e^{1}=3 / 2$ and $e^{2}=2$ instead, then there is an $\varepsilon>0$ that satisfies (SC), and $\omega_{3}$ is preferred by both consumer types, since $U^{1}\left(\omega_{1} ; 1,0,1,3\right)=0.62$, $U^{1}\left(\omega_{3} ; 1,-2.61,1,10\right)=0.90, U^{2}\left(\omega_{2} ; 1,0,1,1\right)=0.35$, and $U^{2}\left(\omega_{3} ; 1,2.61,1,3\right)=0.54$.

${ }^{16}$ For $\omega_{3}$, we choose $t_{\omega_{3}}^{1}$ to maximize $\sum_{\theta=1,2} \tilde{u}^{\theta}\left(x, g_{\omega}, n_{\omega}\right)$ and make $t_{\omega}^{2}=-t_{\omega}^{1}$ (since $n^{1}=$ $\left.n^{2}=1\right)$ and the budget constraints to hold with equality.
} 
in: $t_{\omega}^{\theta}$ (reflecting the consumer's tastes for the public goods in the jurisdiction), $p \tau_{\omega}$ (the cost of financing the local public goods), and $\alpha_{\omega}^{\theta}$ (wage that reflects the consumer's marginal productivity in the jurisdiction). This sort of differentiated pricing might be interesting for future empirical work.

Externalities and spatial considerations: Observe that in contrast to EGSZ (2006) and Zame (2007), we do not consider externalities in the private production, such as poor working conditions (in EGSZ (2006) wages are set to internalize the production externalities). Neither do we consider local consumption externalities within each jurisdiction (or development), as in Konishi (2011). Our main focus here is to analyze the Berglas' "group composition problem" as it was initially conceived, where these types of externalities were not present.

Spatial considerations are absent in our model. However, space could be incorporated by assigning a location to every possible jurisdiction (following Konishi (2008)). In such a spatial Berglas' economy, any two jurisdictions that are sufficiently close geographically may have some residents in one jurisdiction that benefit from the public goods of the other jurisdiction. Such behavior has been avoided in all previous Tiebout literature by assuming that every jurisdiction has the capacity to expel "illegal consumers" ("exclusion"). In this sense, we are also accepting this assumption in order to guarantee efficiency. If this assumption were absent, then the pricing would be inefficient, as it would not capture the externalities imposed by those commuters. Recovering efficiency without the exclusivity assumption is an interesting line of investigation, but is beyond the scope of this paper.

Tastes and skills: Assumption B2 is essential for Theorem 2 to hold true since skills (or endowments) act as an efficient fiscal discriminatory device among consumers, because such characteristics are linked in a one-to-one relationship with the consumers' tastes for public goods. This assumption might be justified in certain cases. For example, one could think that individuals that have been assigned a certain type of job in the industry are prone to demand specific health services. Individuals with different working conditions (say, miners and engineers) will demand different public health services, and thus will be taxed differently. If signaling (through skills or endowments) does not exist, then an equilibrium for this Berglas economy is not efficient. The best hope in such a scenario is a Lindahl pricing scheme. ${ }^{17}$

\footnotetext{
${ }^{17} \mathrm{~A}$ more sophisticated approach could then be taken. In particular, one could seek to make
} 
We observe that Assumption B2 can be relaxed in a novel way.

Assumption B2': Consumers of the same type are assigned the same endowments, skills, and tastes for public goods, but may have different preferences for private goods.

Remark 1: Theorem 1 also holds true under assumption B2'. However, Schmeidler's (1973) purification result can no longer be applied (see discussion below). Also, Theorem 2 holds true if $B \mathscr{2}^{\prime}$ is imposed instead.

On the equilibrium existence technique: We investigate the problem of existence of a price taking equilibrium by transforming it into a problem of existence of a social system equilibrium (Debreu (1952)). Our approach is by simultaneous optimization. As Arrow and Debreu (1954) assert, with a generalized game we are able to test in a clearer way the consistency of the equations that describe the model. In the simultaneous optimization approach each player maximizes a payoff function on a constraint set. Both the payoff function and the constraint set may be parameterized by the other players' actions. This second dependence does not occur in games. The extension is a mathematical object referred to as a generalized game by Debreu (1952). In the generalized game there are auctioneers that mimic the market by choosing the prices optimally in order to make the excess demand zero (if it is positive (negative) for some commodities the auctioneer chooses higher (lower) prices for those goods).

Kakutani: Both the non-excess demand approach and the simultaneous optimization approach are applications of Kakutani's (1941) fixed point theorem. Debreu (1982) clearly exposes the two parallel approaches in a rigorous way. The non-excess demand approach constructs an excess demand correspondence and finds a price vector that makes it zero. To assure this, it uses the theorem of Gale (1955), Nikaido (1956), and Debreu (1956). The simultaneous optimization approach, on the other hand, is an application of the Nash (1950) theorem to a generalized game, known as the Debreu (1952) theorem. Arrow and Debreu (1954) then applied the Debreu (1952) theorem to a social economic system, where agents simultaneously seek to maximize their respective payoff functions. Also it is fair to mention here the paper Shafer and Sonnenschein (1975), which is a more modern version of the Debreu (1952) social existence theorem. Schafer and Sonnenschein proved that a competitive equilibrium exists also for abstract skills an endogenous choice as in Conley and Wooders (2001). We refer to the original paper for such a refinement. 
economies with interdependent, price-dependent and non-ordered preferences.

The continuum extension: The three approaches (core, non excess demand and simultaneous optimization approach) were initially conceived for an exchange economy with a finite number of agents. The extension of the core approach to an atomless local public good economy was done by Kaneko and Wooders (1986). Since then, Wooders and coauthors have further enriched the model in many different ways using the f-core notion. The application of the non-excess demand approach to an atomless club economy was done by EGSZ (1999). For this EGSZ (1999) followed the pioneering works of Aumann (1964, 1966). In the present paper we not only provide the first equilibrium existence proof for a club / local public good economy using a generalized game, but also carry out this analysis in the continuum of agents framework. Most of our extensions follow by application of Hildebrand's (1974) results. It is worth noting that the technique of proving existence of equilibrium in a nonatomic economy using a generalized game has been useful in the incomplete markets literature (see for example, Araujo, Orrillo and Páscoa (2000) and Araujo and Páscoa (2002)). However, this technique has never been applied to a local public goods nonatomic economy.

Purification: Finally, we wish to stress that our purification result also departs from earlier works on club / local public good economies. The discreteness of the set of public projects (in the sense of Ellickson (1979) and Mas-Colell (1980)) requires a continuum of agents in order to convexify agents' strategy sets. However, purification of players' mixed strategies cannot be done using Schmeidler's (1973) result if agents of the same type have the same tastes on public projects, but can differ in their preferences on the private goods (Assumption B2'). This implies that their demands for commodities may be different among consumers of the same type. Instead, we apply a particular result of Páscoa (1998), already used by Araujo and Páscoa (2002, Lemma 2) in an incomplete markets economy, which says that purification can be done if in the extended generalized game we have players' mixed strategies depending only through finitely many indicators, one for each type (a statistic indicator).

\section{Generalized game and proofs}

Theorem 1 is proved assuming that assumption B2' holds. Throughout the proof we discuss how the proof can be modified if assumption B2 applies instead. 
Proof of Theorem 1: We first construct a generalized game for a Berglas' economy and prove that it has an equilibrium. Then, we show that such equilibrium is, in fact, an equilibrium.

The generalized game: In the generalized game a player $k$ chooses his strategy $s(k)$ parameterized by the other agents' strategies $\bar{s}_{-k}$. For this economy the game is played by the consumers and four additional auctioneers. We divide the consumers' optimization problem into two stages.

Stage 1: Consumer $h_{\theta}$ chooses his most preferred consumption bundle for a given jurisdiction membership $m=(\theta, \omega)$ with $\mu^{h_{\theta}}(\theta, \omega)=1$.

$$
\max _{\left\{x_{\omega}\right\}} u^{h_{\theta}}\left(\cdot, \bar{\mu}^{h_{\theta}}(\theta, \omega)\right) \text { s.t. } \bar{p}\left(x_{\omega}-e^{\theta}\right)+\bar{t}_{\omega}^{\theta}+\bar{p} \bar{\tau}_{\omega}-\bar{\alpha}_{\omega}^{\theta} \leq 0
$$

Observe that the terms in $\mathrm{BC}_{h_{\theta}}(\omega)$ are all multiplied by $\bar{\mu}^{h_{\theta}}(\theta, \omega)$, but we omit it as we know that $\bar{\mu}^{h_{\theta}}(\theta, \omega)=1$ (the consumer is evaluating his utility at this specific jurisdiction type). Let us denote consumer $h_{\theta}$ 's demand correspondence for private goods at jurisdiction $\omega$ by $\Psi\left(h_{\theta}, \omega\right) \equiv\left\{\operatorname{argmax} u^{h_{\theta}}\left(\cdot, \bar{\mu}^{h_{\theta}}(\theta, \omega)\right)\right.$ : $\mathrm{BC}_{\omega}\left(h_{\theta}\right)$ holds $\}$. Note that, for a consumer $h_{\theta}$, the correspondence $h_{\theta} \rightarrow \Psi\left(h_{\theta}, \omega\right)$ has a measurable graph (see Hildebrand (1974, p. 59, Proposition 1.b)).

Commodity prices can be shown to be positive. The usual procedure is to build a truncated economy by adjoining a few agents of each type with utilities linear in the consumption of the private good. For this truncated economy, the prices of private goods and memberships belong to compact sets. Using a feasibility argument we can show that commodity prices are positive along the sequence. Then, we can apply Fatou's lemma to show that the sequence of truncated economies has an equilibrium, independent of the truncation (the measure of the set of artificial adjoined agents goes to zero). For simplicity, we omit this procedure and refert to EGSZ (1999).

Claim 1: $\Psi\left(h_{\theta}, \omega\right)$ has nonempty convex compact values and is continuous.

Proof: Compactness follows because $\sum_{\theta} \int_{H(\theta)} e^{\theta} d \lambda<\infty$. By quasiconcavity of $u^{h_{\theta}}$ and convexity of the values of the budget correspondence $B^{h_{\theta}}\left(\bar{p}, \bar{q}_{\omega}^{\theta}, \bar{\tau}_{\omega}, \bar{\alpha}_{\omega}^{\theta}\right.$, $\omega) \equiv\left\{x_{\omega}^{h_{\theta}} \in \mathbb{R}_{+}^{L}: B C\left(h_{\theta}\right)\right.$ holds $\}$, it follows that $\Psi\left(h_{\theta}, \omega\right)$ has convex values. The budget correspondence $B\left(h_{\theta}, \bar{p}, \bar{q}_{\omega}^{\theta}, \bar{\tau}_{\omega}, \bar{\alpha}_{\omega}^{\theta}, \omega\right)$ and the utility function $u^{h_{\theta}}\left(x_{\omega}, \mu^{h_{\theta}}(\theta, \omega)\right)$ are continuous in $x$. By Berge's Maximum theorem and endowment desirability, $\Psi\left(h_{\theta}, \omega\right)$ is upper semi-continuous. Lower semi-continuity of $\Psi\left(h_{\theta}, \omega\right)$ follows by the interiority of endowments assumption. Actually, at nonzero commodity prices the consumer's budget constraint holds with strict 
inequality by choosing a sufficiently small consumption bundle. Thus, $\Psi\left(h_{\theta}, \omega\right)$ is continuous.

Let $\Psi(\theta, \omega)=\int_{H(\theta)} \Psi\left(h_{\theta}, \omega\right) d \lambda$ be a measurable correspondence from the continuum of type $\theta$ consumers at jurisdiction $\omega$ into $\mathbb{R}_{+}^{L}$. Observe that there is a measurable selection $h_{\theta} \rightarrow \psi\left(h_{\theta}, \omega\right)$ from the correspondence $\boldsymbol{\Psi}(\theta, \omega)$, and we can therefore choose an aggregate demand vector $\int_{H(\theta)} \psi\left(h_{\theta}, \omega\right) d \lambda$. Because $\int_{H(\theta)} \psi\left(h_{\theta}, \omega\right) d \lambda$ is the integral of upper semicontinuous demands with respect to a nonatomic measure, we have that $\int_{H(\theta)} \psi\left(h_{\theta}, \omega\right) d \lambda$ is upper semicontinuous, with compact, convex, and nonempty values.

Stage 2: Given their optimal consumption bundles, consumers choose their most preferred jurisdiction type (notice that the consumer being alone in a jurisdiction is a possibility). Let $U^{h_{\theta}}\left(\omega, \bar{p}, \bar{t}_{\omega}^{\theta}, \bar{\tau}_{\omega}, \bar{\alpha}_{\omega}^{\theta}\right) \equiv u^{h_{\theta}}\left(\psi\left(h_{\theta}, \omega\right), \mu^{h_{\theta}}(\theta, \omega)\right)$. Then, $\mu^{h_{\theta}}(\theta, \omega)=1$ for $\omega \in \arg \max U^{h_{\theta}}\left(\omega, \bar{p}, \bar{t}_{\omega}^{\theta}, \bar{\tau}_{\omega}, \bar{\alpha}_{\omega}^{\theta}\right)$. We represent the pure strategy of consumer $h_{\theta}$ by a basis vector of dimension $\Omega$. The vector $\mu^{h_{\theta}}(\theta, \omega)$ is the vector in $\mathbb{R}^{\Omega}$ with 1 as $\omega^{\text {th }}$ coordinate and zero otherwise.

Auctioneer 1 chooses $\alpha=\left(\alpha_{\omega}^{\theta}\right)_{\theta \in \boldsymbol{\Theta}, \omega \in \boldsymbol{\Omega}}$ to $\min _{\{\alpha\}} \sum_{\omega, \theta}\left(\left(\alpha_{\omega}^{\theta}-\bar{p} \frac{\partial y_{\omega}}{\partial \eta_{\omega}^{\theta}}\left(\left(n_{\omega}^{\theta}\right)_{\theta \in \boldsymbol{\Theta}}\right)\right)\right.$ $\left.\int_{H(\theta)} \bar{\mu}^{h_{\theta}}(\theta, \omega) d \lambda\right)^{2}$, where $\frac{\partial y_{\omega}}{\partial \eta_{\omega}^{\theta}}\left(\left(n_{\omega}^{\theta}\right)_{\theta \in \boldsymbol{\Theta}}\right)$ denotes the marginal productivity of a type $\theta$ consumer evaluated at the crowding profile $\left(n_{\omega}^{\theta}\right)_{\theta \in \Theta} \in \mathbb{N}_{+}^{\Theta}$.

Auctioneer 2 chooses a poll fee $\tau_{\omega} \in \mathbb{R}_{+}^{L}$ common to all individuals in the jurisdiction $\left(\tau_{\omega}=\tau_{\omega}^{\theta}, \forall \theta \in \mathbf{\Theta}\right)$ such that it covers the cost of providing the jurisdiction public project, i.e., $\min _{\{\tau\}} \sum_{\omega}\left(\bar{p}\left(\tau_{\omega}-\frac{c\left(g_{\omega}\right)}{n_{\omega}^{\theta}}\right) \sum_{\theta} \int_{H(\theta)} \bar{\mu}^{h_{\theta}}(\theta, \omega) d \lambda\right)^{2}$.

Auctioneer 3 chooses a vector of prices $\left\{t_{\omega}^{\theta}\right\}_{\theta \in \boldsymbol{\Theta}, \omega \in \boldsymbol{\Omega}}$, with $t_{\omega} \in$ Trans, in order to $\min _{\{t\}} \sum_{\omega}\left(\sum_{\theta} t_{\omega}^{\theta} \int_{H(\theta)} \bar{\mu}^{h_{\theta}}(\theta, \omega) d \lambda\right)^{2}$.

Claim 2: Auctioneers 1, 2, and 3's strategy sets are nonempty, convex, and compact.

Proof: Strategy sets are convex since $\alpha_{\omega}^{\theta}, \tau_{\omega}$ and $t_{\omega}^{\theta}$ belong to the real numbers, for all $\theta$ and $\omega$. Transfers are bounded, $t_{\omega}^{\theta} \in\left[T^{-}(\theta), T^{+}(\theta)\right]$. The upper bound is $T^{+}(\theta)=L \max _{l} \sum_{\theta} \int_{H(\theta)} e_{l}^{\theta} d \lambda+\max _{\omega} \sum_{\theta} \int_{H(\theta)} \alpha_{\omega}^{\theta} d \lambda$, since $p \in \Delta^{L-1}$. The term $\sum_{\theta} \int_{H(\theta)} \alpha_{\omega}^{\theta} d \lambda$ is bounded because $y_{\omega} \leq A_{\omega}$, with $A_{\omega} \in(0, \infty)^{L}$, (by Assumption A3(i)). This implies that Auctioneer 1's strategy set is compact. The lower bound on the lump-sum transfers is $T^{-}(\theta)=\min \left\{0, \max _{\omega} \sum_{\theta^{\prime} \neq \theta} n_{\omega}^{\theta^{\prime}} T^{+}\left(\theta^{\prime}\right)\right\}$. The argument is well known. If some consumers are paying large negative lumpsum transfers, then others must be paying large positive lump-sum transfers, which implies that some transfers are canceled with some others (for $t_{\omega} \in$ Trans). 
Hence, Auctioneer 3's strategy set is compact. Finally, notice that poll taxes are bounded since $p c\left(g_{\omega}\right) / n_{\omega}$ is a finite constant component, and $c\left(g_{\omega}\right) \geq 0$ is bounded from above by assumption. Hence, Auctioneer 2's strategy set is compact.

Auctioneer 4 chooses $p$ in $\triangle^{L-1}$ in order to make the aggregate excess demand as expensive as possible, i.e., $\max _{\{p\}} \zeta(p)$, where $\zeta(p) \equiv p\left(\sum_{\theta} \int_{H(\theta)}\left(\bar{x}^{h_{\theta}}-e^{\theta}\right) d \lambda+\right.$ $\left.\sum_{\omega} \gamma(\omega)\left(c\left(g_{\omega}\right)-y_{\omega}\left(\left(n_{\omega}^{\theta}\right)_{\theta \in \boldsymbol{\Theta}}\right)\right)\right)+\sum_{\omega, \theta} \bar{t}_{\omega}^{\theta} \int_{H(\theta)} \bar{\mu}^{h_{\theta}}(\theta, \omega) d \lambda$.

Claim 3: $\zeta(p)$ is bounded below, upper hemicontinuous, and convex-valued.

Proof: The compact-valued correspondence $h_{\theta} \rightarrow \Psi\left(h_{\theta}, \omega ; p\right)$ is bounded below by 0 and above by the integrable function $h_{\theta} \rightarrow\left(\frac{w_{1}}{p_{1}}, \ldots, \frac{w_{L}}{p_{L}}\right)$, where $w_{l}=$ $\sum_{\theta} \lambda(H(\theta)) e_{l}^{\theta}+A_{\omega}, l \in \mathbf{L}$. We have $p_{l}>0, \forall l$. According to Hildebrand (1974, p. 62, Theorem 2), $\int_{H(\theta)} \Psi\left(h_{\theta}, \omega ; p\right) d \lambda \neq \emptyset$. And according to Hildebrand (1974, p. 73, Proposition 7) this set, which is bounded below by 0 , is also compact. Therefore, $\sum_{\theta \in \Theta} \int_{H(\theta)} \psi\left(h_{\theta}, \omega\right) d \lambda$ is nonempty and compact. Upper hemicontinuity of the correspondence $p \rightarrow \Psi\left(h_{\theta}, \omega ; p\right)$ on the interior of the simplex follows by Hildebrand (1974, p. 73, Proposition 8). Therefore, the correspondence $\sum_{\theta \in \Theta} \int_{H(\theta)} \psi\left(h_{\theta}, \omega\right) d \lambda$ is upper hemicontinuous. The correspondence being convex-valued is a consequence of Lyapounov's convexity theorem of an atomless finite dimensional vector measure (see Hildebrand (1974, p. 62, Theorem 3)). By a parallel argument as above, there is a measurable selection $h_{\theta} \rightarrow \mu^{h_{\theta}}(\theta, \omega)$ with an associated aggregate demand vector $\sum_{\theta} \int_{H(\theta)} \mu^{h_{\theta}}(\theta, \omega) d \lambda$, which is the integral of upper semicontinuous demands with respect to a non-atomic measure. Thus, $\sum_{\theta} \int_{H(\theta)} \mu^{h_{\theta}}(\theta, \omega) d \lambda$ is upper semicontinuous, with compact (by the requirement $\sum_{m} \mu^{h_{\theta}}(m)=1$, for a.e. $h_{\theta}$ ), convex (by Lyapounov's convexity theorem) and nonempty values.

Definition GGE: An equilibrium for the constructed generalized game consists of a vector of bundles and memberships $(\bar{x}, \bar{\mu})$ and a vector of prices $(\bar{p}, \bar{t}, \bar{\tau}, \bar{\alpha})$ such that each player $k$ chooses a strategy $s(k)$ to solve his respective optimization problem parameterized in the other players' actions $\bar{s}_{-k}$.

Proposition 2: There exists an equilibrium in mixed strategies for the constructed generalized game.

Proof: Note that a consumer's strategy of choosing his most preferred jurisdiction type in stage 2 has a finite and discrete space domain $\Omega$, since the set $\Theta$ of consumer types, the set $\mathbf{G}$ of public projects, the set $\mathbf{N} \subset \mathbb{N}_{+}^{\Theta}$ of consumers 
profiles, and the set $\mathbf{Y}$ of production technologies are finite (and thus discrete). In order to circumvent this problem, we extend our generalized game to allow for consumers' mixed strategies in the set of jurisdiction types. Let us denote $\Pi(\boldsymbol{\Omega})=\left\{\pi=(\pi(\omega))_{\omega \in \boldsymbol{\Omega}}: \pi(\omega) \geq 0, \sum_{\omega \in \boldsymbol{\Omega}} \pi(\omega)=1\right\}$. Then, $\Pi(\boldsymbol{\Omega})$ stands for the convex hull of $(1, \ldots, \omega, \ldots, \Omega)$, which is the set of mixed strategies for each consumer. A profile of strategies $\rho: \mathbf{H} \rightarrow \Pi(\Omega)$ brings the continuum of consumers into strategies (pure or mixed).

Consumer $h_{\theta}$ 's stage 2 optimization problem extended to mixed strategies is such that this consumer randomizes over the possible consumptions in the different jurisdiction types. We write $U^{h_{\theta}}\left(\pi, \bar{p}, \bar{t}_{\omega}^{\theta}, \bar{\tau}_{\omega}, \bar{\alpha}_{\omega}^{\theta}\right) \equiv u^{h_{\theta}}\left(\sum_{\omega} \pi(\omega) \psi\left(h_{\theta}, \omega\right), \pi\right)$. That is, consumer randomizes in $\Omega=\{1, \ldots, \Omega\}$, but not directly in consumption bundles. Then, consumer $h_{\theta}$ 's stage 2 maximization problem is $\max _{\pi \in \Pi(\boldsymbol{\Omega})} U^{h_{\theta}}(\pi$, $\left.\bar{p}, \bar{t}^{\theta}, \bar{\tau}, \bar{\alpha}^{\theta}\right)$. The utility $u^{h_{\theta}}\left(\sum_{\omega} \pi(\omega) \psi\left(h_{\theta}, \omega\right), \pi\right)$ is a continuous bounded real valued function on $\sum_{\omega} \pi(\omega) \psi\left(h_{\theta}, \omega\right)$, and the mixed strategy $\pi$ belongs to the convex compact set $\Pi(\Omega) . R\left(h_{\theta}\right)=\left\{\pi \in \Pi(\Omega): \pi \in \arg \max U^{h_{\theta}}\left(\pi, \bar{p}, \bar{t} \theta, \bar{\tau}, \bar{\alpha}^{\theta}\right)\right\}$ denotes the set of mixed strategies that solve consumer $h_{\theta}$ 's second stage maximization problem.

We must extent the fictitious auctioneers' problems to allow for consumers' mixed strategies. Given a mixed strategy profile $\rho: \mathbf{H} \rightarrow \Pi(\Omega)$ and the vector of consumers' demand for commodities evaluated in their optimal jurisdiction membership, $\left(\bar{\psi}\left(h_{\theta}, \bar{\mu}^{h_{\theta}}(\theta, \omega)\right)_{h_{\theta} \in \mathbf{H}}\right.$, we can rewrite the continuous linear function of Auctioneer 4 in terms of mixed strategies as follows ${ }^{18}$

$$
p \rightarrow \zeta^{\prime}(p) \equiv \sum_{\theta} \int_{H(\theta)}\left(\left(\int_{\Omega} p \bar{\psi}\left(h_{\theta}, \bar{\mu}^{h_{\theta}}(\theta, \omega)\right)+\left(\bar{t}_{\omega}^{\theta}+p \bar{\tau}_{\omega}-\bar{\alpha}_{\omega}^{\theta}\right)\right) d \rho\left(h_{\theta}\right)-p e^{\theta}\right) d \lambda .
$$

Objective functions $\zeta(p)$ and $\zeta^{\prime}(p)$ are obtained by aggregating consumers' budget constraints. While for $\zeta^{\prime}(p)$ it is in terms of consumers' mixed strategies, $\zeta(p)$ holds for pure strategies. $\zeta(p)$ and $\zeta^{\prime}(p)$ are equivalent in a degenerate equilibrium if consistency holds and profits are zero (we will prove below that consistency and zero profits actually hold in equilibrium).

Auctioneer 1, 2, and 3's objective functions extended to mixed strategies become, respectively,

$$
\begin{gathered}
\alpha \rightarrow \sum_{\omega \in \boldsymbol{\Omega}}\left(\sum_{\theta \in \boldsymbol{\Theta}}\left(\alpha_{\omega}^{\theta}-\bar{p} \frac{\partial y_{\omega}}{\partial \eta_{\omega}^{\theta}}\left(\left(n_{\omega}^{\theta}\right)_{\theta \in \boldsymbol{\Theta}}\right)\right) \int_{H(\theta)} \rho\left(h_{\theta}\right)(\omega) d \lambda\right)^{2}, \\
\tau \rightarrow \sum_{\omega \in \boldsymbol{\Omega}}\left(\bar{p}\left(\tau_{\omega}-\left(c\left(g_{\omega}\right) / n_{\omega}^{\theta}\right)\right) \sum_{\theta \in \boldsymbol{\Theta}} \int_{H(\theta)} \rho\left(h_{\theta}\right)(\omega) d \lambda\right)^{2},
\end{gathered}
$$

\footnotetext{
${ }^{18}$ Observe that for Auctioneer 4's objective function we could have written $\sum_{\omega \in \boldsymbol{\Omega}} \bar{\psi}\left(h_{\theta}, \bar{\mu}^{h_{\theta}}(\theta, \omega) d \rho\left(h_{\theta}\right)(\omega)\right.$ instead of $\int_{\boldsymbol{\Omega}} \bar{\psi}\left(h_{\theta}, \bar{\mu}^{h_{\theta}}(\theta, \omega) d \rho\left(h_{\theta}\right)\right.$.
} 


$$
t \rightarrow \sum_{\omega \in \boldsymbol{\Omega}}\left(\sum_{\theta \in \boldsymbol{\Theta}} t_{\omega}^{\theta} \int_{H(\theta)} \rho\left(h_{\theta}\right)(\omega) d \lambda\right)^{2}
$$

All the conditions of Debreu's (1952) theorem hold. Thus, we can assert that the extended generalized game has an equilibrium, possibly in mixed strategies. At this point it remains to show that a degenerate equilibrium of the extended generalized game is, in fact, an equilibrium of the original game.

Proposition 3: The generalized game has an equilibrium in pure strategies.

Proof: Recall that from Assumption A1 there is a continuum of consumers of each type with positive measure. Observe that Auctioneers 1, 2, and 3's new objective functions depend only on the average of the consumers' profile, which satisfies Schmeidler (1973) hypotheses. However, Schemeidler's hypotheses are not satisfied by Auctioneer 4's payoff function $\zeta^{\prime}(p) .{ }^{19}$ Instead, the conditions in Páscoa (1998) and Araujo and Páscoa' (2002, Lemma 2) are satisfied for this auctioneer's objective function, as it depends on the profile of mixed strategies $\rho=\rho\left(h_{\theta}\right)_{h_{\theta} \in \mathbf{H}}$ only through finitely many indicators, one for each type $\theta$, of the form $\int_{H(\theta)} \int_{\boldsymbol{\Omega}}\left(\bar{\psi}\left(h_{\theta}, \bar{\mu}^{h_{\theta}}(\theta, \omega)\right) d \rho\left(h_{\theta}\right) d \lambda .^{20}\right.$ Given a mixed strategies equilibrium profile $\rho$, there exists a profile $\bar{\mu}=\left(\bar{\mu}\left(h_{\theta}, \omega\right)\right)_{h_{\theta} \in H(\theta), \theta \in \Theta}$ such that the Dirac measure $\hat{\rho}\left(h_{\theta}\right)$ at $\bar{\mu}^{h_{\theta}}(\theta, \omega)$ is an extreme point of the set $R\left(h_{\theta}\right)$, which is consumer $h_{\theta}$ 's best response to the prices chosen by the auctioneers in the previous equilibrium in mixed strategies. Moreover, $\int_{H(\theta)} \int_{\Omega} \bar{\psi}\left(h_{\theta}, \bar{\mu}\left(h_{\theta}, \omega\right)\right) d \rho\left(h_{\theta}\right) d \lambda$ is the same as $\int_{H(\theta)} \int_{\Omega} \bar{\psi}\left(h_{\theta}, \bar{\mu}\left(h_{\theta}, \omega\right)\right) d \hat{\rho}\left(h_{\theta}\right) d \lambda$. Hence, we can replace $\bar{\mu}$ by $\left(\hat{\rho}\left(h_{\theta}\right)\right)_{h_{\theta} \in H(\theta), \theta \in \Theta}$ and keep all the equilibrium conditions satisfied. In fact, once this replacement is done, payoff functions $\zeta(p)$ and $\zeta^{\prime}(p)$ can easily be shown to be equivalent. The indicators that the atomic auctioneer takes as given evaluated at $\hat{\rho}$ are still the same as when they were evaluated at $\rho$. Therefore, $\hat{\rho}$ is a degenerate equilibrium profile.

Notice that if we had assumed instead that consumers of the same type have the same preferences for private goods (Assumption B2), then we would have a common best response $\psi(\theta, \omega)$ instead of one $\psi\left(h_{\theta}, \omega\right)$ for each consumer $h_{\theta}$ of this type. In that case, Schmeidler's (1978) purification result could be applied, since we could write $\int_{H(\theta)} \int_{\Omega} \psi\left(h_{\theta}, \bar{\mu}^{h_{\theta}}(\theta, \omega)\right) d \rho\left(h_{\theta}\right) d \lambda$ as $\int_{\Omega} \psi\left(\theta, \bar{\mu}^{h_{\theta}}(\theta, \omega)\right) d\left(\oint_{H(\theta)}\right.$

\footnotetext{
${ }^{19}$ Schmeidler's (1973) purification result cannot be applied, as it requires a common best demand response for all consumers of the same type.

${ }^{20}$ In fact, our framework is even simpler than the Páscoa (1998) and Araujo and Páscoa (2002, Lemma 2) purification result, since purification has to be done within each type $\theta \in \Theta$ of consumer.
} 
$\left.\rho\left(h_{\theta}\right) d \lambda\right)$, where the term $\oint_{H(\theta)} \rho\left(h_{\theta}\right) d \lambda$ is the Gelfand integral of $\varphi$. In that case, we should check that the market auctioneer's objective function is continuous with respect to the convergence in distribution ${ }^{21}$.

Proposition 4: An equilibrium for the generalized game (in pure strategies) is an equilibrium.

Proof: Let us consider the generalized game introduced above, but where the consumption allocations have an upper bound $\mathbf{U} \subset \mathbb{R}_{+}^{L}$ that exceeds the attainability upper bound by an arbitrary small amount. Let $(\psi, \mu, p, t, \tau, \alpha)$ be an equilibrium in pure strategies of the generalized game.

First notice that Auctioneer 3's minimization problem implies $\sum_{\omega, \theta} t_{\omega}^{\theta} \int_{H(\theta)} \mu^{h_{\theta}}$ $(\theta, \omega) d \lambda=0$. Now, let us show that $\hat{\mu}$ is consistent. If consistency fails, then $\gamma(\omega)$ is such that $\sum_{\theta}\left(t_{\omega}^{\theta}+p \tau_{\omega}-\alpha_{\omega}^{\theta}\right) \gamma(\omega) n_{\omega}^{\theta}<\sum_{\theta}\left(t_{\omega}^{\theta}+p \tau_{\omega}-\alpha_{\omega}^{\theta}\right) \hat{\mu}(\theta, \omega)$. But then, this strict inequality enters into contradiction with the solution to Auctioneer 4's maximization problem.

Observe that the market auctioneer's problem is now $\max _{\{p\}} \zeta^{\prime \prime}(p)$, where $\zeta^{\prime \prime}(p) \equiv p\left(\sum_{\theta, \omega} \int_{H(\theta)}\left(\bar{x}_{\omega}^{h_{\theta}}-e^{\theta}\right) d \lambda+\sum_{\omega} \gamma(\omega)\left(c\left(g_{\omega}\right)-y_{\omega}\left(\left(n_{\omega}^{\theta}\right)_{\theta \in \boldsymbol{\Theta}}\right)\right)\right)$, since $\sum_{\omega, \theta} t_{\omega}^{\theta}$. $\int_{H(\theta)} \mu^{h_{\theta}}(\theta, \omega) d \lambda=0$ (as shown above). We now show that there is no commodity excess demand. Suppose there is excess demand of commodity $l$. Then, the price auctioneer sets $p_{l}$ equal to 1 , but then the whole function $\zeta^{\prime \prime}(p)$ becomes positive, a contradiction with the aggregation of the budget constraints.

Commodities markets clear. Suppose not, that is, $\sum_{\theta, \omega} \int_{H(\theta)}\left(\psi_{l}\left(h_{\theta}, \omega\right)-e_{l}^{\theta}\right) d \lambda$ $+\sum_{\omega} \gamma(\omega)\left(c_{l}\left(g_{\omega}\right)-y_{l, \omega}\left(\left(n_{\omega}^{\theta}\right)_{\theta \in \boldsymbol{\Theta}}\right)\right)<0$ for some $l \in \mathbf{L}$. Then, the market auctioneer chooses $p_{l}$ sufficiently close to zero, which would make $\psi_{l}\left(h_{\theta}\right)$ hit the bound $\mathbf{U}$, for every individual, a contradiction with feasibility.

From Auctioneers 1, 2, and 3's optimization problems in the generalized game, we have $p y_{\omega}\left(\left(n_{\omega}^{\theta}\right)_{\theta \in \boldsymbol{\Theta}}\right)=\sum_{\theta} \alpha_{\omega}^{\theta} n_{\omega}^{\theta}$ and $\sum_{\theta \in \Theta} n_{\omega}^{\theta}\left(t_{\omega}^{\theta}+p \tau_{\omega}\right)=p c\left(g_{\omega}\right), \forall \omega \in \boldsymbol{\Omega}$.

Let us now show that consumers are quasi-optimizing, that is, if $\left(\tilde{x}_{\tilde{\omega}}^{h_{\theta}}, \tilde{\mu}^{h_{\theta}}(\theta, \tilde{\omega})\right)$ $\in \mathbf{X}^{h_{\theta}}$ with $\tilde{x}_{\tilde{\omega}}^{h_{\theta}} \in B^{h_{\theta}}\left(p, q_{\tilde{\omega}}^{\theta}, \tau_{\tilde{\omega}}, \alpha_{\tilde{\omega}}^{\theta}, \tilde{\omega}\right)$ and $u^{h_{\theta}}\left(\tilde{x}_{\tilde{\omega}}^{h_{\theta}}, \tilde{\mu}^{h_{\theta}}(\theta, \tilde{\omega})\right)>u^{h_{\theta}}\left(\psi\left(h_{\theta}, \omega\right), \mu^{h_{\theta}}\right.$ $(\theta, \omega))$, then $p \tilde{x}_{\tilde{\omega}}^{h_{\theta}}+t_{\tilde{\omega}}^{\theta}+p \tau_{\tilde{\omega}}-\alpha_{\tilde{\omega}}^{\theta}-e^{\theta} \geq 0$. Suppose not, that is, such allocation $\left(\tilde{x}_{\omega}^{h_{\theta}}, \tilde{\mu}^{h_{\theta}}(\theta, \tilde{\omega})\right)$ is strictly preferred to $\left(\psi\left(h_{\theta}, \omega\right), \mu^{h_{\theta}}(\theta, \omega)\right)$, and $p \tilde{x}_{\tilde{\omega}}^{h_{\theta}}+t_{\tilde{\omega}}^{\theta}+p \tau_{\tilde{\omega}}-$

\footnotetext{
${ }^{21}$ Call the market auctioneer's objective function $\Lambda$. Convergence in distribution occurs if a sequence of pure strategies profiles $\bar{\mu}^{h_{\theta}}\left(\theta, \omega_{n}\right)$ that converges to $\bar{\mu}^{h_{\theta}}(\theta, \omega)$ is such that $\int_{\mathbf{H}} g\left(\bar{\mu}^{h_{\theta}}\left(\theta, \omega_{n}\right)\right) d \lambda$ converges to $\int_{\mathbf{H}} g\left(\bar{\mu}^{h_{\theta}}(\theta, \omega)\right) d \lambda$, for any bounded continuous transformation $g$ of $\bar{\mu}^{h_{\theta}}(\theta, \omega)$. Then, $\Lambda\left(\bar{\mu}^{h_{\theta}}\left(\theta, \omega_{n}\right)\right)$ converges to $\Lambda\left(\bar{\mu}^{h_{\theta}}(\theta, \omega)\right)$.
} 
$\alpha_{\tilde{\omega}}^{\theta}-e^{\theta}<0$. But then, at such preferred optimum, the aggregation of consumers' budget constraints holds with strict inequality, a contradiction to market clearing and $\sum_{\omega, \theta} t_{\omega}^{\theta} \int_{H(\theta)} \mu^{h_{\theta}}(\theta, \omega) d \lambda=0$.

It remains to check that consumers are optimizing, that is, if $\left(\tilde{x}_{\tilde{\omega}}^{h_{\theta}}, \tilde{\mu}^{h_{\theta}}(\theta\right.$, $\tilde{\omega})) \in \mathbf{X}^{h_{\theta}}$ with $\tilde{x}_{\tilde{\omega}}^{h_{\theta}} \in B^{h_{\theta}}\left(p, q_{\tilde{\omega}}^{\theta}, \tau_{\tilde{\omega}}, \alpha_{\tilde{\omega}}^{\theta}, \tilde{\omega}\right)$ and $u^{h_{\theta}}\left(\tilde{x}_{\tilde{\omega}}^{h_{\theta}}, \tilde{\mu}^{h_{\theta}}(\theta, \tilde{\omega})\right)>u^{h_{\theta}}\left(\psi\left(h_{\theta}, \omega\right)\right.$, $\left.\mu^{h_{\theta}}(\theta, \omega)\right)$, then $p \tilde{x}_{\tilde{\omega}}^{h_{\theta}}+t_{\tilde{\omega}}^{\theta}+p \tau_{\tilde{\omega}}-\alpha_{\tilde{\omega}}^{\theta}-e^{\theta}>0$. We show that a quasi-equilibrium is an equilibrium by contradiction. Suppose that the quasi-equilibrium $((x, \mu),(p, t$, $\alpha, \tau)$ ) is not an equilibrium. Let there be a nonnull set of consumers who are quasioptimizing but not optimizing. For each such consumer $h_{\theta}$, there is a choice $\left(\tilde{x}^{h_{\theta}}, \tilde{\mu}^{h_{\theta}}\right)$ in which $u^{h_{\theta}}\left(\tilde{x}^{h_{\theta}}, \tilde{\mu}^{h_{\theta}}\right)>u^{h_{\theta}}\left(x^{h_{\theta}}, \mu^{h_{\theta}}\right)$ and $p\left(\tilde{x}^{h_{\theta}}-e^{\theta}\right)+t_{\tilde{\omega}}^{\theta}+p \tau_{\tilde{\omega}}-$ $\alpha_{\tilde{\omega}}^{\theta} \leq 0$. Desirability of endowments entails that there exists another bundle $\bar{x}^{h_{\theta}} \leq \tilde{x}^{h_{\theta}}$ with $\bar{x}^{h_{\theta}} \neq \tilde{x}^{h_{\theta}}$ and $\left(\bar{x}^{h_{\theta}}, \tilde{\mu}^{h_{\theta}}\right) \in \mathbf{X}^{h_{\theta}}$, and $\bar{x}^{h_{\theta}} \geq k$ (where $k \geq 0$ stands for the capital requirement of membership $\left.\tilde{\mu}^{h_{\theta}}\right) .{ }^{22}$ By continuity, we can choose the bundle $\bar{x}^{h_{\theta}}$ such that $p \bar{x}^{h_{\theta}}<p \tilde{x}^{h_{\theta}}$ and still have $u^{h_{\theta}}\left(\bar{x}^{h_{\theta}}, \tilde{\mu}^{h_{\theta}}\right)>u^{h_{\theta}}\left(x^{h_{\theta}}, \mu^{h_{\theta}}\right)$, but $p\left(\bar{x}^{h_{\theta}}-e^{\theta}\right)+t_{\tilde{\omega}}^{\theta}+p \tau_{\tilde{\omega}}-\alpha_{\tilde{\omega}}^{\theta}<0$ (so $\left(\bar{x}^{h_{\theta}}, \tilde{\mu}^{h_{\theta}}\right)$ costs strictly less than his quasi-equilibrium choice), a contradiction.

Theorem 1 follows from Propositions 2, 3 and 4 .

Proof of Theorem 2: We first prove that for this Berglas economy any equilibrium $(x, \mu)$ belongs to the core. Suppose not. Then there exists a blocking coalition $\tilde{H} \subseteq \mathbf{H}$ and a feasible allocation $\left(\tilde{x}^{h}, \tilde{\mu}^{h}\right)_{h \in \tilde{H}}$ with $u^{h}\left(\tilde{x}^{h}, \tilde{\mu}^{h}\right) \geq$ $u^{h}\left(x^{h}, \mu^{h}\right)$ for all $h \in \tilde{H} \subset \mathbf{H}$ and $u^{h^{\prime}}\left(\tilde{x}^{h^{\prime}}, \tilde{\mu}^{h^{\prime}}\right)>u^{h^{\prime}}\left(x^{h^{\prime}}, \mu^{h^{\prime}}\right)$ for those consumers $h^{\prime}$ in $H^{\prime} \subset \tilde{H}$ with $\lambda\left(H^{\prime}\right)>0$. Feasibility of $(\tilde{x}, \tilde{\mu})$ implies consistency and market clearing for $\tilde{H}$ and budget balance for all consumers in $\tilde{H}$. Such a feasible and preferred state contradicts the equilibrium, where consumers choose optimally in their budget sets. Therefore, $(x, \mu)$ is in the core of this economy. Pareto efficiency immediately follows by letting $\tilde{H}$ equal the whole population of consumers $\mathbf{H}$.

We now prove that, if in addition Assumption A6 is imposed in our Berglas economy, then any core state $(x, \mu)$ can be supported as an equilibrium. Let us denote the consumer $h_{\theta}$ 's preferred set by $\Phi^{h_{\theta}}=\left\{(\tilde{x}, \iota) \in \mathbf{X}^{h_{\theta}}: u^{h_{\theta}}(\tilde{x}, \iota)>\right.$ $\left.u^{h_{\theta}}\left(x^{h_{\theta}}, \mu^{h_{\theta}}\right)\right\}$. Since a consumer $h_{\theta}$ can choose at most one membership, say $\iota=\iota(\theta, \omega)$ with $|\iota|=1$, we write $\tau(\iota)=c\left(g_{\omega}\right) / n_{\omega}$. Let us be more explicit

\footnotetext{
${ }^{22}$ As EGSZ (2006) point out, this more restricted form of the consumption sets just requires us to be more careful than EGSZ (1999) in the distinction between a quasi-equilibrium and equilibrium.
} 
in notaion and denote the consumer $h_{\theta}$ 's wage function at jurisdiction $\omega$ by $\alpha_{\omega}\left(p, \beta_{\omega}, h_{\theta}\right)$. For each consumer $h_{\theta} \in \mathbf{H}$, let $\sigma^{h_{\theta}}=\left\{(\tilde{x}, \iota) \in \mathbf{X}^{h_{\theta}}:\left(\tilde{x}+e\left(h_{\theta}\right)+\right.\right.$ $\left.\left.\alpha_{\iota}\left(p, \beta_{\omega}, h_{\theta}\right)-\tau_{\iota}, \iota\right) \in \Phi^{h_{\theta}}\right\}$. Finally, define the correspondence $\Sigma^{h_{\theta}}=\sigma^{h_{\theta}} \cup\{0\}$. Observe that the correspondence $\Sigma$ is measurable if the functions $\alpha_{\omega}^{\theta}\left(p, \beta_{\omega}, \cdot\right)$ and $e^{\theta}(\cdot)$ are measurable. The measurability of $e^{\theta}(\cdot)$ is guaranteed by assumption. It remains to check that the function $\alpha_{\omega}\left(p, \beta_{\omega}, \cdot\right)$ is measurable.

By Assumption A6, the mapping $p y_{\omega}(\cdot)$ of $\mathcal{H}_{\omega}$ into $\mathbb{R}_{+}$is countably additive and dominated by $\lambda$ (since $\gamma$ is absolutely continuous with respect to $\lambda$ ). Also, $p y_{\omega}(\emptyset)=0$. Thus, by the theorem of Radon-Nykodyn, there exists a measurable function $\alpha_{\omega}\left(p, \beta_{\omega}, \cdot\right)$ of $\mathcal{H}_{\omega}$ into $\mathbb{R}_{+}$such that $p y_{\omega}\left(\hat{H}_{\omega}\right)=\int_{\hat{H}_{\omega}} \alpha_{\omega}\left(p, \beta_{\omega}, \cdot\right) d \lambda$, for any given set $\hat{H}_{\omega} \in \mathcal{H}_{\omega}$ and any price $p$ (see Hildebrand (1968). This in turn implies the equilibrium condition of no-profits in the production of private goods. Now, observe that the production possibility correspondence $y_{\omega}$ of $\mathcal{H}_{\omega}$ into $\mathbb{R}^{L}$ is compact and convex (by Assumption A3(i) and the finiteness of the measure $\gamma$ ) for every $\hat{H}_{\omega} \in \mathcal{H}_{\omega}$. Also, $\mathbf{0} \in y_{\omega}\left(\hat{H}_{\omega}\right)$ for every $\hat{H}_{\omega} \in \mathcal{H}_{\omega}$ (given our Assumption A3(ii) and $\left.y_{\omega}\left(\hat{H}_{\omega}\right)=\gamma(\omega) y_{\omega}\left(\left(n_{\omega}^{\theta}\right)_{\theta \in \boldsymbol{\Theta}}\right)\right)$. Then, it follows that there exists a Radon-Nykodym derivative of $y_{\omega}\left(\hat{H}_{\omega}\right)$ with respect to measure $\lambda$ (see Hildebrand (1968)). That is, there exists a correspondence $\kappa_{\omega}$ of $\mathcal{H}_{\omega}$ into $\mathbb{R}_{+}^{L}$ such that $Y_{\omega}\left(\hat{H}_{\omega}\right)=\int_{\hat{H}_{\omega}} \kappa_{\omega} d \lambda$ for every $\hat{H}_{\omega} \in \mathcal{H}_{\omega}$.

Now, let $Z=\left(\int_{\mathbf{H}} \Sigma^{h_{\theta}} d \lambda-\sum_{\omega} \int_{\mathbf{H}} \kappa_{\omega} d \lambda, \sum_{\theta} \int_{\mathbf{H}} \tilde{\mu}^{h_{\theta}}(\theta, \omega) d \lambda\right)$. Since the measure space $(\mathbf{H}, \mathcal{H}, \lambda)$ is atomless, it follows by the Lyapounov convexity theorem that $Z$ is a nonempty convex subset of $\mathbb{R}^{L} \times \mathbb{R}^{|\mathbf{M}|}$. (See Hildebrand (1968)). We now show that we can separate $Z$ from a "fat" enough cone $C^{*}$, so that we guarantee $p \neq 0$. The proof of $Z \cap C^{*}=\emptyset$ follows EGSZ (1999, Theorem 5.1, Step 3), where it is shown, by contradiction, that there is no state $(\hat{x}, \hat{\mu})$ preferred to $(x, \mu)$ that is feasible for an "exactly" consistent coalition ${ }^{23}$ whose agents choose in their preferred set. Let us define $D=\inf \{\operatorname{dist}(\operatorname{conv}(\mathcal{L})$, Cons $): \mathcal{L} \in \mathcal{D}\}$, where $\operatorname{conv}(\mathcal{L})$ denotes the convex hull of the set $\mathcal{L}$ and $\mathcal{D}=\{\mathcal{L} \subset$ Lists : $\operatorname{conv}(\mathcal{L}) \cap$ Cons $=\emptyset\}$. We now can construct the "fatter" cone $C^{*}=\{(\bar{x}, \bar{\mu}) \in$ $\mathbb{R}^{L} \times \mathbb{R}^{|\mathbf{M}|}: \bar{x}<-\frac{W}{D} \operatorname{dist}(\bar{\mu}$, Cons $\left.)(1, \ldots, 1)\right\}$. The upper bound on private goods consumption, denoted by $W$, is different from EGSZ (1999), as in our economy we also have to take into account the private goods production. Here, $W=$ $\sum_{\theta} \int_{H(\theta)} e^{\theta} d \lambda+\sum_{\omega} y_{\omega}\left(\hat{H}_{\omega}\right)$, where $\hat{H}_{\omega}=\left\{h \in \mathbf{H}: \bar{\mu}^{h_{\theta}}(\theta, \omega)=1, \forall \theta\right\}$. As shown below, $y_{\omega}\left(\hat{H}_{\omega}\right)<\infty$, for $p>0$. Once we modify this upper bound, the proof of

\footnotetext{
${ }^{23}$ Notice that Lemma 7.1 of EGSZ (1999), which asserts that such exactly consistent coalition can be chosen, does not depend on the bound $W$.
} 
$Z \cap C^{*}=\emptyset$ is analogous to EGSZ (1999).

By Minkowski's separation theorem there exist prices $(p, t) \in \mathbb{R}^{L} \times \mathbb{R}^{|\mathbf{M}|}$, $(p, t) \neq(0,0)$, such that $(p, t)(\bar{x}, \bar{\mu}) \leq 0$ for each $(\bar{x}, \bar{\mu}) \in C^{*}$, and $(p, t) z \geq 0$, for each $z \in Z$. By an argument similar to EGSZ (1999, Theorem 5.1, Step 4), we have $p \neq 0$. The separation of convex cones implies that

$$
p \sum_{\omega} y_{\omega}\left(\hat{H}_{\omega}\right) \leq p \sum_{\theta} \int_{\mathbf{H}}\left(\tilde{x}^{h_{\theta}}-e^{\theta}+\tau_{\omega}\right) d \lambda+\sum_{\omega} t_{\omega} \sum_{\theta} \int_{\mathbf{H}} \tilde{\mu}^{h_{\theta}}(\theta, \omega) d \lambda
$$

Condition (C) implies that $p y_{\omega}\left(\hat{H}_{\omega}\right)<\infty$ for all $\omega$, and thus $\alpha_{\omega}\left(p, \beta_{\omega}, \cdot\right)$ can be chosen as an integrable function of $\hat{H}_{\omega}$ in $\mathbb{R}_{+}$(see Hildebrand (1968)).

We now show that almost every consumer chooses in their budget sets. Denote by $\tau_{\omega}\left(\mu^{h_{\theta}}(\theta, \omega)\right)$ the poll tax paid at jurisdiction $\omega$ when membership $\mu^{h_{\theta}}(\theta, \omega)$. Let $E_{1}=\left\{h \in \mathbf{H}: p\left(x_{\omega}^{h_{\theta}}+\tau_{\omega}\left(\mu^{h_{\theta}}(\theta, \omega)\right)\right)+t_{\omega}^{\theta} \mu^{h_{\theta}}(\theta, \omega)>e^{\theta}+\alpha_{\omega}^{\theta}, \forall \theta\right\}$ and $E_{2}=\left\{h \in \mathbf{H}: p\left(x_{\omega}^{h_{\theta}}+\tau_{\omega}\left(\mu^{h_{\theta}}(\theta, \omega)\right)\right)+t_{\omega}^{\theta} \mu^{h_{\theta}}(\theta, \omega)<e^{\theta}+\alpha_{\omega}^{\theta}, \forall \theta\right\}$. The sets $E_{1}$ and $E_{2}$ are measurable, since endowments and wages are measurable functions. Feasibility of $(x, \mu)$ implies that if $\lambda\left(E_{1}\right)>0$, then $\lambda\left(E_{2}\right)>0$. By a procedure similar to EGSZ (1999, Theorem 5.1, Step 4) we can choose a preferred measurable selection $(\tilde{x}, \tilde{\mu})$ from the correspondence $\Sigma$ so that $p \sum_{\theta} \int_{\mathbf{H}}\left(\tilde{x}^{h_{\theta}}-e^{\theta}+\right.$ $\left.\tau_{\omega}\right) d \lambda+\sum_{\omega} t_{\omega} \sum_{\theta} \int_{\mathbf{H}} \tilde{\mu}^{h_{\theta}}(\theta, \omega) d \lambda<p \sum_{\omega} y_{\omega}\left(\hat{H}_{\omega}\right)$, a contradiction with (C). Thus, $\lambda\left(E_{1}\right)=0$.

Market clearing follows by aggregating consumers' budget constraints in $E_{2}$ and the assumption that utilities are strictly monotonic in the consumption of private goods. Quasi-optimization is proved as in EGSZ (1999, Theorem 5.1, Step 4), so we also omit this proof. Our Lemma 7 above shows that a quasiequilibrium is an equilibrium. This concludes the proof that every core state can be supported as an equilibrium.

Proof of Proposition 1: Let us associate $(\bar{x}, \bar{\mu})$ with $\bar{\omega}$ and $(x, \mu)$ with $\omega$ and $\omega^{\prime}$. Assume that $(x, \mu)$ belongs to the core. Consistency of $\bar{\mu}$ is guaranteed by choosing $\gamma(\bar{\omega}), \gamma(\omega)$ and $\gamma\left(\omega^{\prime}\right)$ such that $\gamma(\bar{\omega})=\gamma(\omega) n_{\omega}^{\theta} / n_{\bar{\omega}}^{\theta}=\gamma\left(\omega^{\prime}\right) n_{\omega^{\prime}}^{\theta^{\prime}} / n_{\bar{\omega}}^{\theta^{\prime}}$. This is easy to see once we observe that $\gamma(\bar{\omega}) n_{\bar{\omega}}^{\theta}=\bar{\mu}(\theta, \bar{\omega})=\lambda(H(\theta))=\mu(\theta, \omega)=$ $\gamma(\omega) n_{\omega}^{\theta}$ and $\gamma(\bar{\omega}) n_{\bar{\omega}}^{\theta^{\prime}}=\bar{\mu}\left(\theta^{\prime}, \bar{\omega}\right)=\lambda\left(H\left(\theta^{\prime}\right)\right)=\mu\left(\theta^{\prime}, \omega^{\prime}\right)=\gamma\left(\omega^{\prime}\right) n_{\omega^{\prime}}^{\theta^{\prime}}$.

Let $\bar{x}^{h_{\theta}}=x^{h_{\theta}}+\varepsilon$, with $\varepsilon=(\varepsilon, \ldots, \varepsilon) \in \mathbb{R}_{++}^{L}$, for every consumer $h_{\theta}$ and every type $\theta$. Without loss of generality, we consider a consumer of type $\theta^{\prime}$. Consumer $h_{\theta^{\prime}}$ 's budget constraints $\mathrm{BC}_{h_{\theta^{\prime}}}(\bar{\omega})$ and $\mathrm{BC}_{h_{\theta^{\prime}}}\left(\omega^{\prime}\right)$ are $\bar{p}\left(\bar{x}^{h_{\theta^{\prime}}}+c\left(g_{\bar{\omega}}\right) / n_{\bar{\omega}}\right)+t_{\bar{\omega}}^{\theta^{\prime}}=$ 
$\bar{p} e^{\theta^{\prime}}+\alpha_{\bar{\omega}}^{\theta^{\prime}}$ and $p\left(x^{h_{\theta^{\prime}}}+c\left(g_{\omega^{\prime}}\right) / n_{\omega^{\prime}}\right)+t_{\omega^{\prime}}^{\theta^{\prime}}=p e^{\theta^{\prime}}+\alpha_{\omega^{\prime}}^{\theta^{\prime}}$, respectively, where $\alpha_{\bar{\omega}}^{\theta^{\prime}}$ is the wage that accommodates in $\mathrm{BC}_{h_{\theta^{\prime}}}(\bar{\omega})$ the consumption increase $\varepsilon$. Substracting $\mathrm{BC}_{h_{\theta^{\prime}}}\left(\omega^{\prime}\right)$ from $\mathrm{BC}_{h_{\theta^{\prime}}}(\bar{\omega})$ and using $\bar{x}^{h_{\theta}}=x^{h_{\theta}}+\varepsilon$ and $t_{\omega^{\prime}}^{\theta^{\prime}}=0$ (since all consumers are homogeneous), we have $\alpha_{\bar{\omega}}^{\theta^{\prime}}-\alpha_{\omega^{\prime}}^{\theta^{\prime}}=\bar{p}\left(x^{h_{\theta^{\prime}}}-e^{\theta^{\prime}}+\varepsilon+c\left(g_{\bar{\omega}}\right) / n_{\bar{\omega}}\right)+t_{\bar{\omega}}^{\theta^{\prime}}-$ $p\left(x^{h_{\theta^{\prime}}}-e^{\theta^{\prime}}+c\left(g_{\omega^{\prime}}\right) / n_{\omega^{\prime}}\right)$.

Using $t_{\bar{\omega}} \in$ Trans, we can write consumer $h_{\theta}$ 's budget constraint in $\bar{\omega}$ as follows, $\bar{p}\left(x^{h_{\theta}}+\varepsilon+c\left(g_{\bar{\omega}}\right) / n_{\bar{\omega}}\right)-\left(n_{\bar{\omega}}^{\theta^{\prime}} / n_{\bar{\omega}}^{\theta}\right) t_{\bar{\omega}}^{\theta^{\prime}}=\bar{p} e^{\theta}+\alpha_{\bar{\omega}}^{\theta}$. Then, substituting $t_{\bar{\omega}}^{\theta^{\prime}}=\left(n_{\bar{\omega}}^{\theta} / n_{\bar{\omega}}^{\theta^{\prime}}\right)\left(\bar{p}\left(x^{h_{\theta}}-e^{\theta}+\varepsilon+c\left(g_{\bar{\omega}}\right) / n_{\bar{\omega}}\right)-\alpha_{\bar{\omega}}^{\theta}\right)$ in the previous equation and removing the negative components that are affected by prices $\bar{p}$ and $p$, we find that constraint $\mathrm{BC}_{h_{\theta^{\prime}}}(\bar{\omega})$ is feasible if wages are such that $n_{\bar{\omega}}^{\theta^{\prime}}\left(\alpha_{\bar{\omega}}^{\theta^{\prime}}-\alpha_{\omega^{\prime}}^{\theta^{\prime}}\right)+n_{\bar{\omega}}^{\theta} \alpha_{\bar{\omega}}^{\theta}>$ $n_{\bar{\omega}}^{\theta^{\prime}} \bar{p} x^{h_{\theta^{\prime}}}+n_{\bar{\omega}}^{\theta} \bar{p} x^{h_{\theta}}+n_{\bar{\omega}} \bar{p}\left(\varepsilon+c\left(g_{\bar{\omega}}\right) / n_{\bar{\omega}}\right)+n_{\bar{\omega}}^{\theta^{\prime}} p e^{\theta^{\prime}}$.

Using the fact that prices belong to the simplex and the fact that $\varepsilon$ and $c\left(g_{\bar{\omega}}\right) / n_{\bar{\omega}}$ are independent vectors with positive coordinates, wages must satisfy the following stricter inequality: $n_{\bar{\omega}}^{\theta^{\prime}}\left(\alpha_{\bar{\omega}}^{\theta^{\prime}}-\alpha_{\omega^{\prime}}^{\theta^{\prime}}\right)+n_{\bar{\omega}}^{\theta} \alpha_{\bar{\omega}}^{\theta}>n_{\bar{\omega}}^{\theta^{\prime}} \max _{l} x_{l}^{h_{\theta^{\prime}}}+$ $n_{\bar{\omega}}^{\theta} \max _{l} x_{l}^{h_{\theta}}+n_{\bar{\omega}}\left(\varepsilon+\max _{l} c_{l}\left(g_{\bar{\omega}}\right) / n_{\bar{\omega}}\right)+n_{\bar{\omega}}^{\theta^{\prime}} \max _{l} e_{l}^{\theta^{\prime}}$. Now, since $p$ belongs to the simplex, $p\left(x^{h_{\theta^{\prime}}}+c\left(g_{\omega^{\prime}}\right) / n_{\omega^{\prime}}\right) \leq \alpha_{\omega^{\prime}}^{\theta^{\prime}}+p e^{\theta^{\prime}}$ implies $x_{l}^{h_{\theta^{\prime}}} \leq \alpha_{\omega^{\prime}}^{\theta^{\prime}}+\max _{l} e_{l}^{\theta^{\prime}}$, for any good $l$. The same is true for a type $\theta$ consumer, that is, $x_{l}^{h_{\theta}} \leq \alpha_{\omega}^{\theta}+\max _{l} e_{l}^{\theta}$, for any good $l$. Then, $n_{\bar{\omega}}^{\theta^{\prime}}\left(\alpha_{\bar{\omega}}^{\theta^{\prime}}-\alpha_{\omega^{\prime}}^{\theta^{\prime}}\right)+n_{\bar{\omega}}^{\theta} \alpha_{\bar{\omega}}^{\theta}>n_{\bar{\omega}}^{\theta^{\prime}}\left(\alpha_{\omega^{\prime}}^{\theta^{\prime}}+\max _{l} e_{l}^{\theta^{\prime}}\right)+n_{\bar{\omega}}^{\theta}\left(\alpha_{\omega}^{\theta}+\max _{l} e_{l}^{\theta}\right)+$ $n_{\bar{\omega}}\left(\varepsilon+\max _{l} c_{l}\left(g_{\bar{\omega}}\right) / n_{\bar{\omega}}\right)+n_{\bar{\omega}}^{\theta^{\prime}} \max _{l} e_{l}^{\theta^{\prime}}$. A bit of algebra shows that $\beta_{\bar{\omega}}^{\theta^{\prime}}\left(\alpha_{\bar{\omega}}^{\theta^{\prime}}-\alpha_{\omega^{\prime}}^{\theta^{\prime}}\right)+$ $\beta_{\bar{\omega}}^{\theta}\left(\alpha_{\bar{\omega}}^{\theta}-\alpha_{\omega}^{\theta}\right)-\beta_{\bar{\omega}}^{\theta^{\prime}} \alpha_{\omega^{\prime}}^{\theta^{\prime}}-2 \beta_{\bar{\omega}}^{\theta^{\prime}} \max _{l} e_{l}^{\theta^{\prime}}-\beta_{\bar{\omega}}^{\theta} \max _{l} e_{l}^{\theta}-\max _{l} c_{l}\left(g_{\bar{\omega}}\right) / n_{\bar{\omega}}>\varepsilon$. For a type $\theta$ consumer, the analogous condition is $\beta_{\bar{\omega}}^{\theta^{\prime}}\left(\alpha_{\bar{\omega}}^{\theta^{\prime}}-\alpha_{\omega^{\prime}}^{\theta^{\prime}}\right)+\beta_{\bar{\omega}}^{\theta}\left(\alpha_{\bar{\omega}}^{\theta}-\alpha_{\omega}^{\theta}\right)-\beta_{\bar{\omega}}^{\theta} \alpha_{\omega}^{\theta}-$ $2 \beta_{\bar{\omega}}^{\theta} \max _{l} e_{l}^{\theta}-\beta_{\bar{\omega}}^{\theta^{\prime}} \max _{l} e_{l}^{\theta^{\prime}}-\max _{l} c_{l}\left(g_{\bar{\omega}}\right) / n_{\bar{\omega}}>\varepsilon . \operatorname{Using} 2 \max \left\{\beta_{\bar{\omega}}^{\theta^{\prime}}, \beta_{\bar{\omega}}^{\theta}\right\} \max _{l, \bar{\theta}} e_{l}^{\bar{\theta}}>$ $2 \beta_{\bar{\omega}}^{\theta} \max _{l} e_{l}^{\theta}+\beta_{\bar{\omega}}^{\theta^{\prime}} \max _{l} e_{l}^{\theta^{\prime}}$, we find that the following condition is sufficient for a consumer of any type $\left(\tilde{\theta}=\theta, \theta^{\prime}\right)$ to increase his consumtion by $\varepsilon$ in state $(\bar{x}, \bar{\mu})$ : $\beta_{\bar{\omega}}^{\theta^{\prime}}\left(\alpha_{\bar{\omega}}^{\theta^{\prime}}-\alpha_{\omega^{\prime}}^{\theta^{\prime}}\right)+\beta_{\bar{\omega}}^{\theta}\left(\alpha_{\bar{\omega}}^{\theta}-\alpha_{\omega}^{\theta}\right)-\max \left\{\beta_{\bar{\omega}}^{\theta} \alpha_{\omega}^{\theta}, \beta_{\bar{\omega}}^{\theta^{\prime}} \alpha_{\omega^{\prime}}^{\theta^{\prime}}\right\}-2 \max \left\{\beta_{\bar{\omega}}^{\theta^{\prime}}, \beta_{\bar{\omega}}^{\theta}\right\} \max _{l, \tilde{\theta}} e_{l}^{\tilde{\theta}}-$ $\max _{l} c_{l}\left(g_{\bar{\omega}}\right) / n_{\bar{\omega}}>\varepsilon$. Let us denote this condition by (SC $\left.\alpha\right)$. Thus, the state $(\bar{x}, \bar{\mu})$ is feasible under wages $\left(\alpha_{\omega}^{\bar{\theta}}\right)_{\bar{\theta}=\theta, \theta^{\prime}}$ and improves upon $(x, \mu)$, contradicting the assumption that a state composed only by homogeneous communities is in the core. Hence, the core must be composed by heterogeneous communities.

In this economy equilibrium exists (guaranteed by Assumptions A1-A5 and B1-B3) and is characterized by heterogeneous communities (Theorem 2 holds since we assumed A6) if condition ( $\mathrm{SC} \alpha$ ) holds. We know that in equilibrium, $\alpha_{\bar{\omega}}^{\bar{\theta}}=\bar{p}\left(\partial y_{\bar{\omega}}\left(n_{\bar{\omega}}^{\theta}, n_{\bar{\omega}}^{\theta^{\prime}}\right) / \partial \eta^{\bar{\theta}}\right)$, for $\bar{\theta}=\theta, \theta^{\prime}$, and $\alpha_{\omega^{\prime}}^{\theta^{\prime}}=p\left(\partial y_{\omega^{\prime}}\left(n_{\omega}^{\theta^{\prime}}\right) / \partial \eta^{\theta^{\prime}}\right)$. Since every jurisdiction industry produces only good, we can normalize the price of this good to 1 , and write $\Delta_{\bar{\omega}, \omega^{\prime}}^{\theta^{\prime}} \equiv \alpha_{\bar{\omega}}^{\theta^{\prime}}-\alpha_{\omega^{\prime}}^{\theta^{\prime}}=\left(\partial y_{\bar{\omega}}\left(n_{\bar{\omega}}^{\theta}, n_{\bar{\omega}}^{\theta^{\prime}}\right) / \partial \eta^{\theta^{\prime}}\right)-\left(\partial y_{\bar{\omega}}\left(n_{\bar{\omega}}^{\theta}, n_{\bar{\omega}}^{\theta^{\prime}}\right) / \partial \eta^{\theta^{\prime}}\right)$ 
and $\Delta_{\bar{\omega}, \omega}^{\theta} \equiv \alpha_{\bar{\omega}}^{\theta}-\alpha_{\omega}^{\theta}=\left(\partial y_{\bar{\omega}}\left(n_{\bar{\omega}}^{\theta}, n_{\bar{\omega}}^{\theta^{\prime}}\right) / \partial \eta^{\theta}\right)-\left(\partial y_{\omega^{\prime}}\left(n_{\omega}^{\theta^{\prime}}\right) / \partial \eta^{\theta}\right)$. Then, a sufficient condition for both types of consumers to increase their consumtion by

$\varepsilon$ in state $(\bar{x}, \bar{\mu})$ is $\beta_{\bar{\omega}}^{\theta^{\prime}} \Delta_{\bar{\omega}, \omega^{\prime}}^{\theta^{\prime}}+\beta_{\bar{\omega}}^{\theta} \Delta_{\tilde{\omega}, \omega}^{\theta}-\max _{(\tilde{\theta}, \tilde{\omega})=(\theta, \omega),\left(\theta^{\prime}, \omega^{\prime}\right)} \beta_{\bar{\omega}}^{\tilde{\theta}}\left(\partial y_{\tilde{\omega}}\left(n_{\tilde{\omega}}^{\tilde{\theta}}\right) / \partial \eta^{\tilde{\theta}}\right)-$ $2 \max \left\{\beta_{\bar{\omega}}^{\theta^{\prime}}, \beta_{\bar{\omega}}^{\theta}\right\} \max _{l, \bar{\theta}} e_{l}^{\bar{\theta}}-\max _{l} c_{l}\left(g_{\bar{\omega}}\right) / n_{\bar{\omega}}>\varepsilon$.

\section{References}

[1] Alesina, A. and E. La Ferrara (2000), "Participation In Heterogeneous Communities", Quart. J. Econ. 115, 847-904.

[2] Allouch, N., J. Conley, and M.H. Wooders (2009), "Anonymous price taking equilibrium in Tiebout economies with a continuum of agents: Existence and characterization", J. Math. Econ. 45, 492-510.

[3] Allouch, N. and M. H. Wooders (2008), "Price Taking Equilibrium in Economies with Multiple Memberships in Clubs and Unbounded Club Sizes", J. Econ. Theory 140, 246-278.

[4] Araujo, A., J. Orrillo and M. Páscoa (2000), "Equilibrium with Default and Endogenous Collateral", Math. Finance 10, 1-21.

[5] Araujo, A. and M. Páscoa (2002), "Bankruptcy in a Model of Unsecured Claims", Econ. Theory 20, 455-481.

[6] Arrow, K. and G. Debreu (1954), "Existence of an Equilibrium For a Competitive Economy", Econometrica 22, 265-289.

[7] Aumann, R.J. (1966), "Existence of Competitive Equilibria in Markets with a Continuum of Traders", Econometrica 34, 1-17.

[8] Aumann, R.J. (1964), "Market with a Continuum of Traders", Econometrica $32,39-50$.

[9] de Bartolome, C. (1990), "Equilibrium and Inefficiency in a Community Model With Peer-Group Effects", J. Polit. Economy 98, 110-133.

[10] Benabou, R. (1993), "Workings of a City: Location, Education and Production", Quart. J. Econ. 105, 619-652.

[11] Berglas, E. (1976), "Distribution of Tastes and Skills and the Provision of Local Public Goods", J. Pub. Econ. 6, 409-423. 
[12] Bewley, T. (1981), "A Critique of Tiebout's Theory of Local Public Expenditures", Econometrica 49, 713-740.

[13] Conley, J. and M.H. Wooders (2001), "Tiebout Economies with Differential Genetic Types and Endogenous Chosen Crowding Characteristics", J. Econ. Theory 98, 261-294.

[14] Conley, J. and M.H. Wooders (1997), "Equivalence of the Core and Competitive Equilibrium in a Tiebout Economy with Crowding Types", J. Urban Econ. 41, 421-440.

[15] Debreu, G. (1982), "Existence of Competitive Equilibrium", in Handbook of Mathematical Economics Vol. II, Chapter 15, in K. Arrow and M. Intriligator, ed.

[16] Debreu, G. (1956), "Market equilibrium", Proceedings of the National Academy of Science of the U.S.A. 42, 876-878.

[17] Debreu, G. (1952), "A Social Equilibrium Existence Theorem", Proceedings of the National Academy of Sciences 38, 886-893.

[18] Ellickson, B. (1979), "Competitive Equilibrium with Local Public Goods", J. Econ. Theory 21, 46-61.

[19] Ellickson, B., B. Grodal, S. Scotchmer, and W.R. Zame (1999), "Clubs and the Market", Econometrica 67, 1185-1217.

[20] Ellickson, B., B. Grodal, S. Scotchmer, and W.R. Zame (2006), "The Organization of Production, Consumption and Learning", in K. Vind, ed. The Birgit Grodal Symposium. Berlin: Springer.

[21] Freeman, S. (1996), "Equilibrium Income Inequality among Identical Agents", J. Polit. Economy 104, 1047-1064.

[22] Gale, D. (1955), "The law of supply and demand", Mathematica Scandinavica 3, 155-169.

[23] Gilles, R. and S. Scotchmer (1997), "Decentralization in Replicated Club Economies with Multiple Private Goods", Econ. Theory 72, 363-387.

[24] Glaeser, E. and A. Saiz (2004), "The Rise of the Skilled City", in BrookingsWharton Pap. on Urban Aff. 5, 47-94. 
[25] Hildebrand, W. (1974), "Core and Equilibrium in a Large Economy", Princeton: Princeton University Press.

[26] Hildebrand, W. (1974), "The Core of an Economy with a Measure Space of Economic Agents", Rev. Econ. Stud. 35, 443-452.

[27] Kakutani, S. (1941), "A generalization of Brower's fixed point theorem", Duke Mathematical Journal 18, 457-459.

[28] Kaneko, M. and M.H. Wooders (1986), "The Core of a Game With a Continuum of Players and Finite Coalitions: The Model and Some Results", Math. Soc. Sci. 12, 105-137.

[29] Konishi, H. (2011), "Entrepreneurial Land Developers: Local Externalities and Mixed Housing Developments", mimeo.

[30] Konishi, H. (2010), "Efficient Mixed Clubs: Nonlinear-Pricing Equilibria with Entreprenerial Managers, Japanese Econ. Rev. 61, 35-63. (2009 JEA Nakahara Prize Lecture).

[31] Konishi, H. (2008), "Tiebout's Tale in Spatial Economies: Entrepreneurship, Self-Selection and Efficiency", Reg. Sci. Urban Econ. 38, 461-471, (special issue in honor of Masahisa Fujita).

[32] Luque, J. (2009), "Essays in Equilibrium Theory: Repo Markets and Formation of Jurisdictions with Public Goods", Doctoral dissertation at Universidade Nova de Lisboa, Portugal.

[33] Mas-Colell, A. (1980), "Efficiency and Decentralization in the Pure Theory of Public Goods", Quart. J. Econ. 94, 625-641.

[34] McGuire, M. (1991), "Group Composition, Collective Consumption and Collaborative Production", Amer. Econ. Rev. 81, 1391-140\%.

[35] Mookherjee, D. and D. Ray (2010), "Inequality and Markets: Some Implications of Occupational Diversity", American Economic Journal: Microeconomics 2, 38-76.

[36] Nash, J. (1950), "Equilibrium points in N-person games", Proceedings of the National Academy of Science of the U.S.A. 36, 48-49.

[37] Nikaido, H. (1956), "On the classical multilateral exchange problem", Metronomica 8, 135-145. 
[38] Páscoa, M. (1998), "Nash Equilibrium and the Law of Large Numbers", Int. J. Game Theory 27, 83-92.

[39] Pauly, M.V. (1970), "Cores and Clubs", Public Choice 14, 53-65.

[40] Roback J. (1982), "Wages, Rents and the Quality of Life", J. Polit. Economy 90, 1257-1278.

[41] Rosen, S. (1974), "Hedonic Prices and Implicit Markets: Product Differenciation in Pure Competition", J. Polit. Economy 82, 34-55.

[42] Schmeidler, D. (1973), "Equilibrium Points of Non-atomic Games", Journal Statistical Physics 7, 295-301.

[43] Schwab, R. and W. Oates (1991), "Community Composition and the Provision of Local Public Goods: A Normalized Analysis", J. Pub. Econ. 44, 217-237.

[44] Scotchmer, S. and M.H. Wooders (1987), "Competitive Equilibria and the Core in Economies with Anonymous Crowding", J. Pub. Econ. 34, 159-174.

[45] Shafer, W. and H. Sonnenschein, (1975), "Equilibrium in abstract economies without ordered preferences", J. Math. Econ. 2, 345-348.

[46] Starrett, D. (1988), "Foundations of Public Economics", Cambridge University Press: Cambridge, U.K.

[47] Tiebout, C. (1956), "A Pure Theory of Local Public Expenditures", J. Polit. Economy 64, 416-424.

[48] Wooders, M.H. (1978), "Equilibria, the Core, and Jurisdiction Structures in Economies with a Local Public Good", J. Econ. Theory 18, 328-348.

[49] Zame, W.R. (2007), "Incentives, Contracts and Markets: A General Equilibrium Theory of Firms", Econometrica 75, 1453-1500. 